\title{
Constraint-based probabilistic modeling for statistical abduction
}

\author{
Taisuke Sato $\cdot$ Masakazu Ishihata $\cdot$ Katsumi Inoue
}

Received: 17 September 2009 / Revised: 17 June 2010 / Accepted: 5 July 2010 /

Published online: 19 August 2010

(C) The Author(s) 2010

\begin{abstract}
We introduce a new framework for logic-based probabilistic modeling called constraint-based probabilistic modeling which defines CBPMs (constraint-based probabilistic models), i.e. conditional joint distributions $P(\cdot \mid K B)$ over independent propositional variables constrained by a knowledge base $K B$ consisting of clauses. We first prove that generative models such as PCFGs and discriminative models such as CRFs have equivalent CBPMs as long as they are discrete. We then prove that CBPMs in infinite domains exist which give existentially closed logical consequences of $K B$ probability one. Finally we derive an EM algorithm for the parameter learning of CBPMs and apply it to statistical abduction.
\end{abstract}

Keywords Probabilistic model · Constraint · Abduction

\section{Introduction}

Suppose we have i.i.d. data as a bag of ground atoms and wish to build their logic-based probabilistic model (Getoor and Taskar 2007; De Raedt and Kersting 2008). Theoretically there are many ways to do it but current approaches seem classified into two types, feature-based discriminative approaches and rule-based generative approaches. The former type defines a log-linear model $P(x)=Z^{-1} \exp \left(\sum_{i} w_{i} f_{i}(x)\right)$ where the $f_{i}$ 's are "features", i.e. real-valued functions returning in the case of Boolean ones 1 (true) or 0 (false),

Editors: Hendrik Blockeel, Karsten Borgwardt, Luc De Raedt, Pedro Domingos, Kristian Kersting, Xifeng Yan.

T. Sato $(\bowtie) \cdot$ M. Ishihata

Tokyo Institute of Technology, 2-12-1, Ookayama, Meguro-ku, Tokyo, Japan

e-mail: sato@mi.cs.titech.ac.jp

M. Ishihata

e-mail: ishihata@mi.cs.titech.ac.jp

K. Inoue

National Institute of Informatics, 2-1-2, Hitotsubashi, Chiyoda-ku, Tokyo, Japan

e-mail: kii@nii.ac.jp 
the $w_{i}$ 's weights and $Z$ a normalizing constant. For example CFDs (case-factor diagrams) (McAllester et al. 2004) adopt Boolean features to define a distribution over a "feasible" set of truth assignments $x$ for Boolean variables. MLNs (Markov logic networks) (Richardson and Domingos 2006) use first-order clauses as features that count the number of clauses' ground instances which are true in the Herbrand interpretation $x$.

Contrastingly the latter type, rule-based approaches such as SLPs (Muggleton 1996), ICL (Poole 1997), PRISM (Sato and Kameya 2001, 2008) and more recently ProbLog (De Raedt et al. 2007), employ logical rules, i.e. definite or general clauses to describe a probabilistic data generation process. They proof-theoretically define a distribution over ground atoms (Muggleton 1996; Poole 1997), or model-theoretically define a probability measure on possible worlds, i.e. the set of Herbrand interpretations (Sato and Kameya 2001; De Raedt et al. 2007). Joint distributions thus defined are a subclass of log-linear models where the normalizing constant is unity but able to represent a variety of probabilistic models from BNs (Bayesian networks) to PCFGs (probabilistic context free grammars).

In this paper $^{1,2}$ we introduce constraint-based probabilistic modeling, a new modeling framework which deals with the above two types uniformly. It defines CBPMs (constraintbased probabilistic models), i.e. conditional joint distributions, or more generally conditional probability measures $P_{c}(\cdot \mid K B)^{3}$ on the Herbrand interpretations for $K B$ such that $P_{c}(\cdot)$ is a finite or infinite product of Bernoulli distributions and $K B$ is a set of propositional or first-order clauses. It is motivated by an observation that it is difficult for the current rule-based approaches to deal with some kind of logical knowledge including disjunctive knowledge such as win(rock) $\vee$ win(paper) $\vee$ win(scissors), looping rules such as friend $(x, y) \Leftarrow \operatorname{friend}(y, x)$ and cyclic causal chains such as chemical reactions among metabolites in a metabolic network (Chen et al. 2008). We wish to logically express those types of knowledge by CBPMs using arbitrary clauses in probabilistic modeling and apply logical inference and statistical inference together.

The basic idea of CBPMs is simple: independent propositional variables (ground atoms) are constrained by a knowledge base $K B$ so that their joint distribution is the intended one. To illustrate it, let us take the simplest example and consider a distribution $P(X=x)$ for a single random variable $X$ that takes on values $\{a, b\}$. We represent $P(X=x)$ as a CBPM as follows. Introduce a propositional variable $\lceil X=a\rceil$ corresponding to the event $X=a$ together with probability $P_{c}(\lceil X=a\rceil)=\frac{P(X=a)}{1+P(X=a)}$ and similarly for $\lceil X=b\rceil$, where $P_{c}(\cdot)$ is a joint distribution that makes $\lceil X=a\rceil$ and $\lceil X=b\rceil$ independent. Since either $X=a$ or $X=b$ always happens but they never happen together, we impose a logical constraint, exclusive-or, $K B_{X}=(\lceil X=a\rceil \vee\lceil X=b\rceil) \wedge \neg(\lceil X=a\rceil \wedge\lceil X=b\rceil)$ on them. Then we see

$$
\begin{aligned}
& P_{c}\left(\lceil X=a\rceil \mid K B_{X}\right) \\
& \quad=\frac{P_{c}\left(\lceil X=a\rceil \wedge K B_{X}\right)}{P_{c}\left(K B_{X}\right)} \\
& =\frac{P_{c}(\lceil X=a\rceil) P_{c}(\neg\lceil X=b\rceil)}{P_{c}(\lceil X=a\rceil) P_{c}(\neg\lceil X=b\rceil)+P_{c}(\neg\lceil X=a\rceil) P_{c}(\lceil X=b\rceil)}
\end{aligned}
$$

\footnotetext{
${ }^{1}$ This paper is a revised version of a conference paper presented at ILP 2009 with the same title augmented with proofs for theorems and new sections for infinite CBPMs, related work and a new learning experiment.

${ }^{2}$ Distributions are discrete throughout the paper.

${ }^{3}$ A joint distribution $P\left(X_{1}=x_{1}, \ldots, X_{n}=x_{n}\right)$ is a probability measure on $\mathbf{R}^{n}$ induced by random variables $X_{1}, \ldots, X_{n}$. When $n$ is countably infinite, the distribution is considered as a probability measure on $\mathbf{R}^{\infty}$. To deal with finite $n$ and infinite $n$ uniformly, when the context is clear, we use "joint distribution" and "probability measure" synonymously.
} 


$$
\begin{aligned}
& =\frac{P(X=a)}{P(X=a)+P(X=b)} \\
& =P(X=a) .
\end{aligned}
$$

Similarly $P_{c}\left(\lceil X=b\rceil \mid K B_{X}\right)=P(X=b)$ holds. In this way $P(X=x)$ is representable as a CBPM $P_{c}\left(\lceil X=x\rceil \mid K B_{X}\right)(x \in\{a, b\})$.

This example is trivial but by developing the idea behind it, we can show that CBPMs are expressive and powerful. That is, they can express both generative models such as PCFGs and discriminative models such as CRFs (conditional random fields) (Lafferty et al. 2001). In addition, we can represent our first-order knowledge in a knowledge base $K B$ and perform logical inference freely. When domains (Herbrand universes) are finite, we can prove that logically equivalent $K B$ s define the same distribution, and hence we may replace one $K B$ with another as long as they are logically equivalent, which would be difficult for featurebased approaches that use logical formulas as features. ${ }^{4}$ Also we allow any types of clause in $K B$ be they non-Horn ones or looping ones that might cause an infinite loop in logic programs. Furthermore despite CBPMs' broad coverage of probabilistic models, their probabilities are uniformly computed and learned from data by the EMC algorithm described in Sect. 4.

Primary advantages of CBPMs from the viewpoint of knowledge representation are generality covering generative and discriminative models and logical expressiveness due to the use of first-order clauses. The treatment of infinite domains is another advantage: CBPMs are always definable even for infinite domains and for arbitrary $K B$ s. From a machine learning point of view, what is new is the EMC algorithm, an EM algorithm applicable to discrete (binarized) conditional distributions with hidden variables.

In what follows, after introducing CBPMs, we prove basic theorems in Sect. 2. Section 3 defines CBPMs in infinite domains. In Sect. 4, we get back to finite domains and derive the EMC algorithm for the parameter learning of CBPMs. In Sect. 5, we apply CBPMs to constraint-based statistical abduction. Section 6 contains related work and Sect. 7 is conclusion.

\section{Constraint-based probabilistic models}

\subsection{Introducing CBPMs}

Let $\mathcal{L}$ be a countable first order language, $\mathcal{U}_{\mathcal{H}}$ the Herbrand universe, i.e. the set of ground terms in $\mathcal{L}$ and $\mathcal{B}_{\mathcal{H}}$ the Herbrand base, i.e. the set of ground atoms in $\mathcal{L}$. We fix an enumeration $A_{1}, A_{2}, \ldots$ of ground atoms in $\mathcal{B}_{\mathcal{H}}$ and identify a $0-1$ vector such as $(1,0, \ldots)$ with a Herbrand interpretation, i.e. truth assignment for $\mathcal{B}_{\mathcal{H}}$ such that $A_{1}=1$ (true), $A_{2}=0$ (false) ... If it makes a closed formula $\varphi$ true, it is called a Herbrand model of $\varphi$. We use the Cartesian product $\mathcal{I}_{\mathcal{H}}=\prod_{i}\{0,1\}_{i}$ to denote the set of all Herbrand interpretations. We assume each $\{0,1\}_{i}$ representing the truth values of $A_{i}$ is a discrete probability space with a probability measure $\mu_{i}(\cdot)$.

\footnotetext{
${ }^{4}$ Consider a non-ground unit clause $P(x)$ representing $\forall x P(x)$. In the Herbrand universe $\{a, b\}$, it is logically equivalent to $P(a) \wedge P(b)$ or to the clause set $\{P(a), P(b)\}$, but $\{P(x)\},\{P(a) \wedge P(b)\}$ and $\{P(a), P(b)\}$ are different feature sets. Likewise $\{A, A \Rightarrow B\}$ and $\{A, B\}$ are logically equivalent but different Boolean feature sets. When we replace logically equivalent but different feature sets, we need to adjust parameters (weights) to preserve the distribution.
} 
Let $P_{c}(\cdot)$ be a product probability measure of the $\mu_{i}$ 's on $\mathcal{I}_{\mathcal{H}}$. We consider $A_{i}$ as a binary random variable from $\mathcal{I}_{\mathcal{H}}$ to $\{0,1\}$ such that $A_{i}(\omega)=x_{i}$ for $\omega=\left(x_{1}, x_{2}, \ldots\right) \in \mathcal{I}_{\mathcal{H}}$ with $P_{c}\left(A_{i}=x_{i}\right)=\mu_{i}\left(\left\{x_{i}\right\}\right)\left(x_{i} \in\{0,1\}\right) . P_{c}(\cdot)$ makes all ground atoms independent and every closed formula $\varphi$ in $\mathcal{L}$ is a binary random variable such that $\varphi(\omega)=1$ if $\omega \models \varphi$ else 0 for $\omega \in \mathcal{I}_{\mathcal{H}}$ with the probability $P_{c}(\varphi=1)=P_{c}\left(\left\{\omega \in \mathcal{I}_{\mathcal{H}} \mid \omega \models \varphi\right\}\right)$. We write $P_{c}(\varphi)$ (resp. $\left.P_{c}(\neg \varphi)\right)$ instead of $P_{c}(\varphi=1)$ (resp. $\left.P_{c}(\varphi=0)\right)^{5}$ and $P(x)$ instead of $P(X=x)$ when the context is clear. We use $V(X)$ for the set of values a random variable $X$ takes.

A $C B P M$ (constraint-based probabilistic model) is a conditional probability measure $P_{c}(\cdot \mid K B)$ on the Herbrand interpretations $\mathcal{I}_{\mathcal{H}}$ where $K B$ is a set of countably many clauses. We assume $K B$ is consistent. Although $P_{c}(\varphi \mid K B)$, the conditional probability of a closed formula $\varphi$, is definable measure-theoretically for any $K B$, when $P_{c}(K B)=0$, we are unable to define it as $\frac{P_{c}(\varphi \wedge K B)}{P_{c}(K B)}$. So hereafter, to make probability computation feasible and discussion simple, we assume, unless otherwise stated, that $\mathcal{L}$ has no function symbol, $\mathcal{B}_{\mathcal{H}}$ is finite and $P_{c}(K B)>0$ (see Sect. 3 for the infinite case).

Consider a joint distribution $P\left(X_{1}=x_{1}, \ldots, X_{N}=x_{N}\right)$ and a CBPM $P_{c}\left(\left\lceil X_{1}=\right.\right.$ $\left.\left.x_{1}\right\rceil, \ldots,\left\lceil X_{N}=x_{N}\right\rceil \mid K B\right)$ where the $\left\lceil X_{i}=x_{i}\right\rceil$ 's are arbitrary propositional variables (ground atoms) ${ }^{6}$ such that $\left\lceil X_{i}=x_{i}\right\rceil$ uniquely corresponds to the event $X_{i}=x_{i}\left(x_{i} \in\right.$ $\left.V\left(X_{i}\right), 1 \leq i \leq N\right)$. When $P_{c}\left(\left\lceil X_{1}=x_{1}\right\rceil, \ldots,\left\lceil X_{N}=x_{N}\right\rceil \mid K B\right)=P\left(X_{1}=x_{1}, \ldots, X_{N}=\right.$ $\left.x_{N}\right)$ holds for every $x_{i}\left(x_{i} \in V\left(X_{i}\right), 1 \leq i \leq N\right),{ }^{7}$ we say that $P_{c}\left(\left\lceil X_{1}=x_{1}\right\rceil, \ldots,\left\lceil X_{N}=\right.\right.$ $\left.\left.x_{N}\right\rceil \mid K B\right)$ is equivalent to $P\left(X_{1}=x_{1}, \ldots, X_{N}=x_{N}\right)$. We prove a basic theorem on CBPMs.

Theorem 1 Every joint distribution has an equivalent CBPM.

Proof Let $P\left(X_{1}=x_{1}, \ldots, X_{N}=x_{N}\right)$ be a joint distribution. We correspondingly introduce state variables $\left\lceil X_{i}=x_{i}\right\rceil$ and parameter variables $\theta_{x_{1}, \ldots, x_{N}}\left(x_{i} \in V\left(X_{i}\right), 1 \leq i \leq N\right)$, and define $K B$ as follows.

$$
\begin{aligned}
X O R\left(X_{i}\right) & =\left(\bigvee_{x_{i} \in V\left(X_{i}\right)}\left\lceil X_{i}=x_{i}\right\rceil\right) \wedge \bigwedge_{x_{i} \neq x_{i}^{\prime}} \neg\left(\left\lceil X_{i}=x_{i}\right\rceil \wedge\left\lceil X_{i}=x_{i}^{\prime}\right\rceil\right), \\
X O R & =\bigwedge_{i=1}^{N} X O R\left(X_{i}\right), \\
E Q U & =\bigwedge_{x_{1}, \ldots, x_{N}}\left(\bigwedge_{i=1}^{N}\left\lceil X_{i}=x_{i}\right\rceil \Leftrightarrow \theta_{x_{1}, \ldots, x_{N}}\right), \\
K B & =X O R \wedge E Q U .
\end{aligned}
$$

\footnotetext{
${ }^{5}$ This applies to a set of formulas as well.

${ }^{6}$ Propositional variables and ground atoms are synonymous in this paper.

${ }^{7}$ We sometimes use vector notation such as $P(\boldsymbol{X}=\boldsymbol{x})$ when non-ambiguous which denotes $P\left(X_{1}=\right.$ $\left.x_{1}, \ldots, X_{N}=x_{N}\right)$ where $\boldsymbol{X}=\left(X_{1}, \ldots, X_{N}\right)$ and $\boldsymbol{x}=\left(x_{1}, \ldots, x_{N}\right)$. 
$X O R\left(X_{i}\right)$ says that $X_{i}$ exclusively takes on one of the values in $V\left(X_{i}\right) . K B$ is equivalent to the following DNF formula where disjuncts are mutually exclusive.

$$
\begin{aligned}
K B \Leftrightarrow & \bigvee_{x_{1}, \ldots, x_{N}}\left\{\bigwedge_{i=1}^{N}\left(\left\lceil X_{i}=x_{i}\right\rceil \wedge \bigwedge_{x_{i}^{\prime} \neq x_{i}} \neg\left\lceil X_{i}=x_{i}^{\prime}\right\rceil\right)\right. \\
& \left.\wedge(\overbrace{x_{1}, \ldots, x_{N}} \wedge \bigwedge_{\left(x_{1}, \ldots, x_{N}\right) \neq\left(x_{1}^{\prime}, \ldots, x_{N}^{\prime}\right)} \neg \theta_{x_{1}^{\prime}, \ldots, x_{N}^{\prime}})\right\} .
\end{aligned}
$$

Next introduce a joint distribution $P_{c}(\cdot)$ which makes all the variables hitherto introduced independent such that

$$
\begin{aligned}
P_{c}\left(\left\lceil X_{1}=x_{1}\right\rceil, \ldots,\left\lceil X_{N}=x_{N}\right\rceil, \theta_{x_{1}, \ldots, x_{N}}\right) & =\left(\prod_{i=1}^{N} P_{c}\left(\left\lceil X_{i}=x_{i}\right\rceil\right)\right) P_{c}\left(\theta_{x_{1}, \ldots, x_{N}}\right), \\
P_{c}\left(\left\lceil X_{i}=x_{i}\right\rceil\right) & =1 / 2 \quad \text { for } \forall i, x_{i} \in V\left(X_{i}\right), \\
P_{c}\left(\theta_{x_{1}, \ldots, x_{N}}\right) & =\frac{P\left(X_{1}=x_{1}, \ldots, X_{N}=x_{N}\right)}{1+P\left(X_{1}=x_{1}, \ldots, X_{N}=x_{N}\right)} .
\end{aligned}
$$

It holds that $0 \leq P_{c}\left(\theta_{x_{1}, \ldots, x_{N}}\right) \leq 1 / 2$ and $P\left(X_{1}=x_{1}, \ldots, X_{N}=x_{N}\right)=\frac{P_{c}\left(\theta_{x_{1}, \ldots, x_{N}}\right)}{P_{c}\left(\neg \theta_{x_{1}}, \ldots, x_{N}\right)}$. We see, by calculation,

$$
\begin{aligned}
P_{c}(K B) & =\alpha \sum_{x_{1}, \ldots, x_{N}} \prod_{i=1}^{N}\left(\frac{P_{c}\left(\left\lceil X_{i}=x_{i}\right\rceil\right)}{P_{c}\left(\neg\left\lceil X_{i}=x_{i}\right\rceil\right)}\right)\left(\frac{P_{c}\left(\theta_{x_{1}, \ldots, x_{N}}\right)}{P_{c}\left(\neg \theta_{x_{1}, \ldots, x_{N}}\right)}\right) \\
& =\alpha \sum_{x_{1}, \ldots, x_{N}}\left(\frac{P_{c}\left(\theta_{x_{1}, \ldots, x_{N}}\right)}{P_{c}\left(\neg \theta_{x_{1}, \ldots, x_{N}}\right)}\right)=\alpha \sum_{x_{1}, \ldots, x_{N}} P\left(X_{1}=x_{1}, \ldots, X_{N}=x_{N}\right) \\
& =\alpha
\end{aligned}
$$

where

$$
\alpha=\left(\prod_{i=1}^{N} \prod_{x_{i}} P_{c}\left(\neg\left\lceil X_{i}=x_{i}\right\rceil\right)\right) \prod_{x_{1}, \ldots, x_{N}} P_{c}\left(\neg \theta_{x_{1}, \ldots, x_{N}}\right)>0 .
$$

Hence we conclude

$$
\begin{aligned}
& P_{c}\left(\left\lceil X_{1}=x_{1}\right\rceil, \ldots,\left\lceil X_{N}=x_{N}\right\rceil \mid K B\right) \\
& \quad=\frac{\left(\alpha \frac{P_{c}\left(\theta_{x_{1}, \ldots, x_{N}}\right)}{P_{c}\left(\neg \theta_{x_{1}, \ldots, x_{N}}\right)}\right)}{\alpha}=\frac{P_{c}\left(\theta_{x_{1}, \ldots, x_{N}}\right)}{P_{c}\left(\neg \theta_{x_{1}, \ldots, x_{N}}\right)} \\
& =P\left(X_{1}=x_{1}, \ldots, X_{N}=x_{N}\right) .
\end{aligned}
$$

\subsection{CBPMs for BNs}

Theorem 1 is general and applicable to BNs. However if applied to a $\mathrm{BN}$ defining a joint distribution $P\left(X_{1}=x_{1}, \ldots, X_{N}=x_{N}\right)=\prod_{i=1}^{N} P\left(X_{i}=x_{i} \mid \Pi_{i}=\pi_{i}\right)$ where $\Pi_{i}$ is the random vector consisting of parent variables of $X_{i}$ and $\pi_{i}$ is its value, we need as many as 
$\left|V\left(X_{1}\right) \times \cdots \times V\left(X_{N}\right)\right|$ parameter variables, which is exponential in $N$. A better way to construct an equivalent but more compact CBPM is to respect the factorized structure of the $\mathrm{BN}$ and to encode CPTs (conditional probability tables) $P\left(X_{i}=x_{i} \mid \Pi_{i}=\pi_{i}\right)$ individually following (Chavira and Darwiche 2005). They introduce Boolean formulas $E Q U_{i}$ and $\operatorname{XOR}\left(X_{i}\right)^{8}(1 \leq i \leq N)$ where

$$
\begin{aligned}
E Q U_{i} & =\bigwedge_{x_{i}, \pi_{i}}\left(\left\lceil X_{i}=x_{i}\right\rceil \wedge\left\lceil\Pi_{i}=\pi_{i}\right\rceil \Leftrightarrow \theta_{x_{i} \mid \pi_{i}}\right), \\
K B_{\mathrm{BN}} & =\bigwedge_{i=1}^{N} X O R\left(X_{i}\right) \wedge E Q U_{i}
\end{aligned}
$$

and compile the conjunction, $K B_{\mathrm{BN}}$, to an $\mathrm{AC}$ (arithmetic circuit) that can compute arbitrary marginal probabilities of the original BN. Since no joint distribution is given to the Boolean variables in their approach, they are not random variables. However, using the above encoding, we can prove the equivalence as follows. First put $P_{c}\left(\left\lceil X_{i}=x_{i}\right\rceil\right)=1 / 2$ and $P_{c}\left(\theta_{x_{i} \mid \pi_{i}}\right)=\frac{P\left(X_{i}=x_{i} \mid \Pi_{i}=\pi_{i}\right)}{1+P\left(X_{i}=x_{i} \mid \Pi_{i}=\pi_{i}\right)}$ for $\forall i, x_{i}$ and $\pi_{i}$ similarly to Theorem 1 . We have

$$
\begin{aligned}
P_{c}\left(K B_{\mathrm{BN}}\right) & =\alpha_{\mathrm{BN}} \sum_{x_{1}, \ldots, x_{N}} \prod_{i=1}^{N}\left(\frac{P_{c}\left(\theta_{x_{i} \mid \pi_{i}}\right)}{P_{c}\left(\neg \theta_{x_{i} \mid \pi_{i}}\right)}\right) \\
& =\alpha_{\mathrm{BN}} \sum_{x_{1}, \ldots, x_{N}} \prod_{i=1}^{N} P\left(X_{i}=x_{i} \mid \Pi_{i}=\pi_{i}\right) \\
\text { where } \alpha_{\mathrm{BN}} & =\prod_{i=1}^{N}\left(\prod_{x_{i}} P_{c}\left(\neg\left\lceil X_{i}=x_{i}\right\rceil\right)\right)\left(\prod_{x_{i}, \pi_{i}} P_{c}\left(\neg \theta_{x_{i} \mid \pi_{i}}\right)\right)
\end{aligned}
$$

and consequently

$$
\begin{aligned}
P_{c} & \left(\left\lceil X_{1}=x_{1}\right\rceil, \ldots,\left\lceil X_{N}=x_{N}\right\rceil \mid K B_{\mathrm{BN}}\right) \\
& =\frac{\prod_{i=1}^{N} P\left(X_{i}=x_{i} \mid \Pi_{i}=\pi_{i}\right)}{\sum_{x_{1}, \ldots, x_{N}} \prod_{i=1}^{N} P\left(X_{i}=x_{i} \mid \Pi_{i}=\pi_{i}\right)} \\
& =P\left(X_{1}=x_{1}, \ldots, X_{N}=x_{N}\right) .
\end{aligned}
$$

Thus the number of parameter variables $\theta_{x_{i} \mid \pi_{i}}$ required by this construction is the same as the number of parameters in the original $\mathrm{BN}$.

We have two comments on $K B_{\mathrm{BN}}$. First even if a $\mathrm{BN}$ has a cyclic directed path, we can construct $K B_{\mathrm{BN}}$ and define $P_{c}\left(\left\lceil X_{1}=x_{1}\right\rceil, \ldots,\left\lceil X_{N}=x_{N}\right\rceil \mid K B_{\mathrm{BN}}\right)$ as long as CPTs are assigned to all nodes. So it can define a joint distribution for cyclic BNs as well as for acyclic BNs. In the cyclic case, the equation (1) tells us that the defined distribution is obtained by normalizing the product of CPTs.

Second, one might imagine $\mathrm{BN}$ probability computation by way of $K B_{\mathrm{BN}}$ is inefficient, but Chavira and Darwiche showed the opposite (Chavira and Darwiche 2005). They demonstrated that this logical encoding outperforms the standard junction tree algorithm by a wide

\footnotetext{
${ }^{8} X O R\left(X_{i}\right)$ is given in the proof of Theorem 1.
} 
margin when optimizations exploiting "local structure" (and subsequent processing to the AC formula) are performed. CBPMs thus can be computationally efficient, if the model class and a computation mechanism are appropriate.

\subsection{CBPMs for log-linear models}

Having seen CBPMs are a general scheme for representing (discrete) joint distributions, we consider two special cases. The first one is log-linear models where a joint distribution $P(\boldsymbol{X}=\boldsymbol{x})$ is given as a product of potential functions $P(\boldsymbol{X}=\boldsymbol{x})=Z^{-1} \prod_{i=1}^{M} F_{i}\left(\boldsymbol{x}_{i}\right)$ $\left(\bigcup_{i=1}^{M} \boldsymbol{x}_{i}=\boldsymbol{x}\right)$ such that $F_{i}\left(\boldsymbol{x}_{i}\right)$ is non-negative and not identically zero. ${ }^{10}$ Here $\boldsymbol{X}, \boldsymbol{x}$ and $\boldsymbol{x}_{i}(\subseteq \boldsymbol{x})$ are vectors and $Z$ is a normalizing constant. In this subsection we show that loglinear models have an equivalent CBPM with the same factorization. The basic strategy is to syntactically separate potential functions and let them generate outputs independently but filter out incompatible ones by equality constraints defined below.

Let us consider a joint distribution $P(X=a, Y=b, Z=c) \propto F_{1}(a, b) F_{2}(b, c)$. To find an equivalent CBPM, we introduce a factor distribution $Q^{(1)}\left(X=a, Y_{1}=b\right)=\frac{F_{1}(a, b)}{\sum_{a, b} F_{1}(a, b)}$ and its equivalent CBPM $P_{c}^{(1)}\left(\lceil X=a\rceil,\left\lceil Y_{1}=b\right\rceil \mid K B_{1}\right)$ as in Theorem 1. Likewise introduce another factor distribution $Q^{(2)}\left(Y_{2}=b, Z=c\right)$ by normalizing $F_{2}(b, c)$ and an equivalent CBPM $P_{c}^{(2)}\left(\left\lceil Y_{2}=b\right\rceil,\lceil Z=c\rceil \mid K B_{2}\right)=Q^{(2)}\left(Y_{2}=b, Z=c\right)=\frac{F_{2}(b, c)}{\sum_{b, c} F_{2}(b, c)} . Y_{1}$ and $Y_{2}$ are syntactic variants of $Y$ in the original distribution $P(X=a, Y=b, Z=c)$. So $V(Y)=V\left(Y_{1}\right)=V\left(Y_{2}\right)$ holds. They are called connection variables for $Y$. Next construct a product distribution $P_{c}=P_{c}^{(1)} \times P_{c}^{(2)}$ such that $P_{c}^{(1)}$ and $P_{c}^{(2)}$ are marginal distributions of $P_{c}$. By simple calculation, we find that

$$
\begin{aligned}
P(X=a, Y=b, Z=c) & \frac{F_{1}(a, b) F_{2}(b, c)}{\sum_{a, b, c} F_{1}(a, b) F_{2}(b, c)}=\frac{Q^{(1)}\left(X=a, Y_{1}=b\right) Q^{(2)}\left(Y_{2}=b, Z=c\right)}{\sum_{a, b, c} Q^{1}\left(X=a, Y_{1}=b\right) Q^{(2)}\left(Y_{2}=b, Z=c\right)} \\
= & \frac{P_{c}^{(1)}\left(\lceil X=a\rceil,\left\lceil Y_{1}=b\right\rceil \mid K B_{1}\right) P_{c}^{(2)}\left(\left\lceil Y_{2}=b\right\rceil,\lceil Z=c\rceil \mid K B_{2}\right)}{\sum_{a, b, c} P_{c}^{(1)}\left(\lceil X=a\rceil,\left\lceil Y_{1}=b\right\rceil \mid K B_{1}\right) P_{c}^{(2)}\left(\left\lceil Y_{2}=b\right\rceil,\lceil Z=c\rceil \mid K B_{2}\right)} \\
& K B_{1} \text { and } K B_{2} \text { have no variable in common and independent w.r.t. } P_{c} \\
= & \frac{P_{c}\left(\lceil X=a\rceil \wedge\left\lceil Y_{1}=b\right\rceil \wedge\left\lceil Y_{2}=b\right\rceil \wedge\lceil Z=c\rceil \wedge K B_{1} \wedge K B_{2}\right)}{P_{c}\left(\bigvee_{a, b, c}\left(\lceil X=a\rceil \wedge\left\lceil Y_{1}=b\right\rceil \wedge\left\lceil Y_{2}=b\right\rceil \wedge\lceil Z=c\rceil \wedge K B_{1} \wedge K B_{2}\right)\right)} \\
= & \frac{P_{c}\left(\lceil X=a\rceil \wedge\left\lceil Y_{1}=b\right\rceil \wedge\left\lceil Y_{2}=b\right\rceil \wedge\lceil Z=c\rceil \wedge K B_{1} \wedge K B_{2}\right)}{P_{c}\left(\bigvee_{a}\lceil X=a\rceil \wedge \bigvee \bigvee_{b}\left(\left\lceil Y_{1}=b\right\rceil \wedge\left\lceil Y_{2}=b\right\rceil\right) \wedge \bigvee \bigvee_{c}\lceil Z=c\rceil \wedge K B_{1} \wedge K B_{2}\right)} \\
& K B_{1} \Rightarrow \bigvee_{a}\lceil X=a\rceil, K B_{2} \Rightarrow \bigvee\lceil Z=c\rceil \text { and }\left\lceil Y_{1}=b\right\rceil \wedge\left\lceil Y_{2}=b\right\rceil \Rightarrow\left\lceil Y_{1}=Y_{2}\right\rceil
\end{aligned}
$$

\footnotetext{
${ }^{9}$ One is determinism, meaning some CPT entries have zero probability. Another is CSI (context-specific independence (Boutilier et al. 1996)) which is value-wise conditional independence.

${ }^{10}$ Usually log-linear models are written as $P(\boldsymbol{x})=Z^{-1} \exp \left(\sum_{i=1}^{M} w_{i} f_{i}\left(\boldsymbol{x}_{i}\right)\right)$ which is always positive. We however prefer a more general form $P(\boldsymbol{x})=Z^{-1} \prod_{i=1}^{M} \exp \left(w_{i} f_{i}\left(\boldsymbol{x}_{i}\right)\right)=\prod_{i=1}^{M} F_{i}\left(\boldsymbol{x}_{i}\right)$ and allow $F_{i}\left(\boldsymbol{x}_{i}\right)$ to be zero at some $\boldsymbol{x}_{i}$.
} 
are tautologies where

$$
\begin{aligned}
\left\lceil Y_{1}=Y_{2}\right\rceil & =\bigvee_{b}\left(\left\lceil Y_{1}=b\right\rceil \wedge\left\lceil Y_{2}=b\right\rceil\right) \\
& =P_{c}\left(\lceil X=a\rceil,\left\lceil Y_{1}=b\right\rceil,\left\lceil Y_{2}=b\right\rceil,\lceil Z=c\rceil \mid\left\lceil Y_{1}=Y_{2}\right\rceil \wedge K B_{1} \wedge K B_{2}\right) \\
& =P_{c}\left(\lceil X=a\rceil,\left\lceil Y_{1}=b\right\rceil,\lceil Z=c\rceil \mid\left\lceil Y_{1}=Y_{2}\right\rceil \wedge K B_{1} \wedge K B_{2}\right) .
\end{aligned}
$$

Thus $P(X=a, Y=b, Z=c) \propto F_{1}(a, b) F_{2}(b, c)$ is expressed as a CBPM. The point of this transformation is the introduction of connection variables $Y_{1}$ and $Y_{2}$ and the addition of an equality constraint $\left\lceil Y_{1}=Y_{2}\right\rceil$ on them to ensure that they take a compatible value $b$ appearing in $F_{1}(a, b)$ and $F_{2}(b, c)$.

Generalizing this example is straightforward. To state Theorem 2 below, we make our terminology precise. Let $P(\boldsymbol{X}=\boldsymbol{x}) \propto \prod_{i=1}^{M} F_{i}\left(\boldsymbol{x}_{i}\right)$ be a given distribution. If $z$, a value of a random variable $Z \in \boldsymbol{X}$, is shared by two or more potential functions $F_{i_{1}}\left(\boldsymbol{x}_{i_{1}}\right), \ldots, F_{i_{k}}\left(\boldsymbol{x}_{i_{k}}\right)$ $(k>1), Z$ is said to be shared. For such $Z$ we introduce new variables $Z_{i_{1}}^{\prime}, \ldots, Z_{i_{k}}^{\prime}$ called connection variables for $Z$ together with a Boolean formula $\bigvee_{z \in V(Z)}\left\lceil Z_{i_{1}}^{\prime}=z\right\rceil \wedge \cdots \wedge\left\lceil Z_{i_{k}}^{\prime}=\right.$ $z\rceil$ called the equality constraint associated with $Z$. Now we have (proof omitted)

Theorem 2 Suppose $P(\boldsymbol{X}=\boldsymbol{x})=Z^{-1} \prod_{i=1}^{M} F_{i}\left(\boldsymbol{x}_{i}\right)\left(\bigcup_{i=1}^{M} \boldsymbol{x}_{i}=\boldsymbol{x}\right)$ is given. Then $P(\boldsymbol{X}=$ $\boldsymbol{x})$ has an equivalent $C B P M P_{c}\left(\left\lceil\boldsymbol{X}_{1}^{\prime}=\boldsymbol{x}_{1}\right\rceil, \ldots,\left\lceil\boldsymbol{X}_{M}^{\prime}=\boldsymbol{x}_{M}\right\rceil \mid C \wedge K B\right)$ with the same factorization as $P$ :

$$
\begin{aligned}
P(\boldsymbol{X}=\boldsymbol{x}) & =P_{c}\left(\left\lceil\boldsymbol{X}_{1}^{\prime}=\boldsymbol{x}_{1}\right\rceil, \ldots,\left\lceil\boldsymbol{X}_{M}^{\prime}=\boldsymbol{x}_{M}\right\rceil \mid C \wedge K B\right) \\
& =\frac{\prod_{i=1}^{M} P_{c}\left(\left\lceil\boldsymbol{X}_{i}^{\prime}=\boldsymbol{x}_{i}\right\rceil \mid K B_{i}\right)}{\sum \boldsymbol{x} \prod_{i=1}^{M} P_{c}\left(\left\lceil\boldsymbol{X}_{i}^{\prime}=\boldsymbol{x}_{i}\right\rceil \mid K B_{i}\right)}
\end{aligned}
$$

where $C$ is a conjunction of the equality constraints associated with shared variables in $\boldsymbol{X}, P_{c}\left(\left\lceil\boldsymbol{X}_{i}^{\prime}=\boldsymbol{x}_{i}\right\rceil \mid K B_{i}\right)$ is a CBPM equivalent to the factor distribution $Q^{(i)}\left(\boldsymbol{X}_{i}^{\prime}=\boldsymbol{x}_{i}\right)=$ $\frac{F_{i}\left(\boldsymbol{x}_{i}\right)}{\sum \boldsymbol{x}_{i} F_{i}\left(\boldsymbol{x}_{i}\right)}(1 \leq i \leq M)$ and $K B=\bigwedge_{i=1}^{M} K B_{i}$.

Here $\lceil\cdot\rceil$ is extended to vector equations in an obvious way. The denominator in (2) is the probability that independent sampling from factor distributions $Q^{(i)}\left(\boldsymbol{X}_{i}^{\prime}=\boldsymbol{x}_{i}\right)(1 \leq i \leq M)$ returns "compatible" values $\boldsymbol{x}_{i}$ such that $\bigcup_{i=1}^{M} \boldsymbol{x}_{i}=\boldsymbol{x}$ for some $\boldsymbol{x}$.

Theorem 2 gives us a way of viewing log-linear models such as CRFs and MLNs as conditional distributions, which leads to a new parameter learning algorithm presented in Sect. 4.

\subsection{Generative models and CBPMs}

We next consider rule-based generative models such as PCFGs. To show that CBPMs can deal with them, we use PRISM which is a symbolic-statistical modeling language based on Prolog extended with a built-in predicate $\mathrm{msw} / 3$ representing probabilistic choices (Sato and Kameya 2001, 2008). PRISM covers generative models in general and PCFGs in particular as exemplified in (Sato and Kameya 2001). We first review PRISM's semantics (distribution semantics) for self-containedness.

A PRISM program $D B=R \cup F$ consists of a set $R$ of definite clauses and a set $F$ of ground $\mathrm{msw}$ atoms. No clause in $R$ contains the $\mathrm{msw}$ predicate in the head. We give a base 
distribution $P_{\mathrm{msw}}(\cdot)$ over the Herbrand interpretations of $F$ in such a way that $\operatorname{msw}(i, t, v)$ represents a probabilistic choice $i$ from exclusive alternatives $V_{i}$ at trial $t$ returning a value $v$ $\left(\in V_{i}\right)$ where $i, t$ and $v$ are arbitrary ground terms. So when $\left\{\operatorname{msw}(i, t, v) \mid v \in V_{i}\right\}$ are drawn from $P_{\mathrm{msw}}(\cdot)$, exactly one of them becomes true for each $i, t$.

$P_{\mathrm{msw}}(\cdot)$ is extended to $P_{D B}(\cdot)$, a probability measure on the set of possible Herbrand interpretations for $D B$, by way of the least model semantics (Lloyd 1984) combined with Kolmogorov's extension theorem as in (Sato and Kameya 2001). $P_{D B}(\cdot)$ turns every closed formula $\varphi$ into a binary random variable having a probability $P_{D B}(\varphi)$. What is important in the distribution semantics is that iff $(R)$, the if-and-only-if completion of $R$ (Lloyd 1984), has probability one, i.e. $P_{D B}($ iff $(R))=1$.

When $\mathcal{B}_{\mathcal{H}}$ is finite as assumed here, iff $(R)$ is given as a Boolean formula iff ${ }^{g}(R) \stackrel{\text { def }}{=}$ $\bigwedge_{H} \operatorname{iff}^{g}(H)$ where $\operatorname{iff}^{g}(H)=H \Leftrightarrow B_{1} \vee \cdots \vee B_{M}(M \geq 0)$ is a formula such that $H$ is a ground atom which is not an msw atom and $\left\{H \Leftarrow B_{1}, \ldots, H \Leftarrow B_{M}\right\}$ is the set of ground clauses from $R$ having $H$ in the head. When $M=0, \operatorname{iff}^{g}(H)=\neg H$. Suppose $G$ is a non-msw ground atom. An explanation for $G$ is a conjunction $E$ of msw atoms in $F$ such that $E \wedge R \vdash G$. We say $G$ has a disjunctive explanation $E_{1} \vee \cdots \vee E_{k}$ if there are explanations $E_{1}, \ldots, E_{k}$ for $G$ satisfying iff ${ }^{g}(R) \vdash G \Leftrightarrow E_{1} \vee \cdots \vee E_{k} .{ }^{11}$ Since $P_{D B}(G)=P_{\mathrm{msw}}\left(E_{1} \vee \cdots \vee E_{k}\right)$ holds thanks to $P_{D B}\left(\right.$ iff $\left.^{g}(R)\right)=1$ by the distribution semantics, we compute $P_{D B}(G)$ by logically reducing $G$ to its disjunctive explanation using iff ${ }^{g}(R)$. It is however generally hard to tell when $G$ has a disjunctive explanation. To state a sufficient condition, we introduce a binary relation " $\succ$ " over $\mathcal{B}_{\mathcal{H}}$ by $A \succ B$ if-andonly-if $B$ appears in the body $W$ of some ground clause $A \Leftarrow W$ from $D B$. $D B$ is said to be cycle-free if there is no looping chain $A_{1} \succ A_{2} \succ \cdots \succ A_{1}$. Then it is rather easy to see that if $D B$ is cycle-free and $\mathcal{B}_{\mathcal{H}}$ is finite, $G$ has a disjunctive explanation such that iff $^{g}(R) \vdash G \Leftrightarrow E_{1} \vee \cdots \vee E_{k}$.

Now let $A_{1}, \ldots, A_{N}$ be an enumeration of non-msw atoms in $\mathcal{B}_{\mathcal{H}}$ ( $\mathcal{B}_{\mathcal{H}}$ is finite). Construct a product $P_{1}$ of Bernoulli distributions over $A_{1}, \ldots, A_{N}$ such that $P_{1}\left(A_{1}\right)=\cdots=P_{1}\left(A_{N}\right)=$ $1 / 2$. Then construct a CBPM $P_{2}$ for msw atoms following the proof of Theorem 1 such that $P_{2}\left(\cdot \mid X O R_{\mathrm{msw}}\right)=P_{\mathrm{msw}}(\cdot)$ holds where $X O R_{\mathrm{msw}}$ is a Boolean formula expressing the mutual exclusiveness and exhaustiveness of msw atoms. Finally define $P_{c}$ as the product distribution of $P_{1}$ and $P_{2}$. Note that the $A_{i}$ 's and the msw atoms are independent w.r.t. $P_{c}$.

Lemma 1 Suppose $\mathcal{B}_{\mathcal{H}}$ is finite and $D B$ is cycle-free. Let $\Delta$ be a Boolean formula consisting of msw atoms. We have $P_{c}\left(\right.$ iff $\left.{ }^{g}(R) \wedge \Delta\right)=(1 / 2)^{N} P_{2}(\Delta)$ for some $N$.

Proof Write iff ${ }^{g}(R)=\bigwedge_{i=1}^{N}\left(A_{i} \Leftrightarrow W_{i}\right)$. Take the transitive closure of $\succ$ and extend it to a total ordering " $\succ^{*}$ " (possible because $\mathcal{B}_{\mathcal{H}}$ is finite and $D B$ is cycle-free). Without loss of generality, we may assume $A_{1} \succ^{*} \cdots \succ^{*} A_{N}$. Note that $A_{1}$ does not occur in $W_{1}$ or in any other $A_{i} \Leftrightarrow W_{i}(i \geq 2)$ because $A_{1}$ is the highest atom in the " $\succ^{*}$ " ordering. So $A_{1}$ is independent of $W_{1}, A_{i} \Leftrightarrow W_{i}(i \geq 2)$ and $\Delta$ w.r.t. $P_{c}$. Put $\Delta^{\prime}=\left(\bigwedge_{i=2}^{N} A_{i} \Leftrightarrow W_{i}\right) \wedge \Delta$. We have

$$
\begin{aligned}
P_{c}\left(\operatorname{iff}^{g}(R) \wedge \Delta\right) & =P_{c}\left(\left(A_{1} \Leftrightarrow W_{1}\right) \wedge \Delta^{\prime}\right) \\
& =P_{c}\left(A_{1} \wedge W_{1} \wedge \Delta^{\prime}\right)+P_{c}\left(\neg A_{1} \wedge \neg W_{1} \wedge \Delta^{\prime}\right) \\
& =1 / 2 P_{c}\left(W_{1} \wedge \Delta^{\prime}\right)+1 / 2 P_{c}\left(\neg W_{1} \wedge \Delta^{\prime}\right)
\end{aligned}
$$

\footnotetext{
${ }^{11}$ Intuitively $G$ is a sentence, explanations $E_{1}, \ldots, E_{k}$ are sentence derivations and iff ${ }^{g}(R)$ is the set of CFG rules used to parse the sentence.
} 


$$
\begin{aligned}
& =1 / 2 P_{c}\left(\left(W_{1} \vee \neg W_{1}\right) \wedge \Delta^{\prime}\right)=1 / 2 P_{c}\left(\Delta^{\prime}\right) \\
& =\cdots=(1 / 2)^{N} P_{c}(\Delta)=(1 / 2)^{N} P_{2}(\Delta) .
\end{aligned}
$$

Theorem 3 Suppose $\mathcal{B}_{\mathcal{H}}$ is finite and a PRISM program $D B$ is cycle-free. Then $D B$ has an equivalent $C B P M$ such that for a non-msw ground atom $G, P_{D B}(G)=P_{c}\left(G \mid\right.$ iff $^{g}(R) \wedge$ $\left.X O R_{\mathrm{msw}}\right)$ where $P_{D B}(G)$ is the probability of $G$ defined by $D B$.

Proof Let $E_{1} \vee \cdots \vee E_{k}$ be a disjunctive explanation for $G . P_{D B}(G)=P_{\mathrm{msw}}\left(E_{1} \vee \cdots \vee E_{k}\right)$ holds. Since iff ${ }^{g}(R) \vdash G \Leftrightarrow E_{1} \vee \cdots \vee E_{k}$, we have, applying Lemma 1,

$$
\begin{aligned}
P_{c}\left(G \mid \text { iff }^{g}(R) \wedge X O R_{\mathrm{msw}}\right) & =P_{c}\left(E_{1} \vee \cdots \vee E_{k} \mid \text { iff }^{g}(R) \wedge X O R_{\mathrm{msw}}\right) \\
& =\frac{P_{c}\left(\left(E_{1} \vee \cdots \vee E_{k}\right) \wedge \text { iff }^{g}(R) \wedge X O R_{\mathrm{msw}}\right)}{P_{c}\left(\text { iff }^{g}(R) \wedge X O R_{\mathrm{msw}}\right)} \\
& =\frac{(1 / 2)^{N} P_{2}\left(\left(E_{1} \vee \cdots \vee E_{k}\right) \wedge X O R_{\mathrm{msw}}\right)}{(1 / 2)^{N} P_{2}\left(X O R_{\mathrm{msw}}\right)} \\
& =P_{2}\left(E_{1} \vee \cdots \vee E_{k} \mid X O R_{\mathrm{msw}}\right)=P_{\mathrm{msw}}\left(E_{1} \vee \cdots \vee E_{k}\right) \\
& =P_{D B}(G) .
\end{aligned}
$$

Theorem 3 assumes $\mathcal{B}_{\mathcal{H}}$ is finite, or equally the number of propositional variables (ground atoms) is finite. As a result Theorem 3 is applicable to BNs but not to PCFGs which require infinitely many random variables. This is theoretically correct but we do not use infinitely many random variables in practice. Think of a PCFG in Chomsky normal form. The backbone CFG rules expressed as a PRISM program take the form $A(i, j) \Leftarrow B(i, j) \wedge C(j, k)$ and $A(i, i+1) \Leftarrow \operatorname{word}(i, i+1)$ where $i, j, k$ are position indexes (Sato and Kameya 2001). When we parse a corpus by the PCFG, only finitely many rules are used because the corpus is finite. Accordingly Theorem 3 is applicable to this finite fragment of the original PCFG, which is practically enough to compute and learn probabilities from the corpus.

Theorem 1 shows that CBPMs are general. Theorem 2 and Theorem 3 show that they are not only able to represent but to "simulate"12 a large class of discrete probabilistic models despite their conceptual simplicity. In addition, probabilities can be efficiently computed based on BDDs (binary decision diagrams) as explained in Sect. 4. In Sect. 5, we exploit the generality of CBPMs to build probabilistic models for abductive reasoning. We conclude this section with logical properties of CBPMs in finite domains.

Proposition 1 Let $P_{c}(\cdot \mid K B)$ be a CBPM conditioned on a Boolean formula KB. Suppose $P_{c}(\cdot)$ gives every Herbrand model of $K B$ a positive probability. ${ }^{13}$ Then for any Boolean formula $\varphi$, it holds that $P_{c}(\varphi \mid K B)=1$ if-and-only-if $K B \vdash \varphi$. It also holds that $P_{c}(\varphi \mid$ $K B)=P_{c}\left(\varphi \mid K B^{\prime}\right)$ if $\vdash K B \Leftrightarrow K B^{\prime}$.

Proof Since $K B \vdash \varphi$ implies $P_{c}(\varphi \mid K B)=1$ is evident, we prove the other way around. Suppose $K B \nvdash \varphi$. So $K B \cup\{\neg \varphi\}$ has a Herbrand model $\omega_{0}$ such that $\omega_{0} \models K B \wedge \neg \varphi$.

\footnotetext{
${ }^{12}$ By "simulate" we mean the preservation of factorization in the case of log-linear models shown in Theorem 2 and goal-subgoal simulation by iff ${ }^{g}(R)$ of SLD derivation for logic programs in the case of rule-based approaches shown in Theorem 3 .

${ }^{13}$ For example, $0<P_{C}(A)<1$ for every ground atom $A$ is enough.
} 
By assumption $P_{c}\left(\left\{\omega_{0}\right\}\right)>0$. It follows that $P_{c}(K B \wedge \neg \varphi)=P_{c}(\{\omega \mid \omega \models K B \wedge \neg \varphi\}) \geq$ $P_{c}\left(\left\{\omega_{0}\right\}\right)>0$. Hence $P_{c}(\varphi \mid K B)=1-P_{c}(\neg \varphi \mid K B)<1$. Also suppose $\vdash K B \Leftrightarrow K B^{\prime}$. Then

$$
P_{c}(\varphi \mid K B)=\frac{P_{c}(\varphi \wedge K B)}{P_{c}(K B)}=\frac{P_{c}\left(\varphi \wedge K B^{\prime}\right)}{P_{c}\left(K B^{\prime}\right)}=P_{c}\left(\varphi \mid K B^{\prime}\right) .
$$

Thus in finite domains, we may replace one $K B$ with another when they are logically equivalent. Also $P_{c}(\varphi \mid K B)=1$ coincides with the notion of logical consequence $K B \models \varphi$. However in infinite domains, the latter is false as we see next.

\section{CBPMs in infinite domains}

\subsection{Conditional probability}

So far we have only been dealing with CBPMs constructed from finitely many Boolean variables (ground atoms) whereas infinite domains such as natural numbers are excluded. In this section, we investigate the differences between CBPMs in finite domains and those in infinite domains. We assume $\mathcal{L}$, the first-order language we use, has function symbols. Then the Herbrand universe $\mathcal{U}_{\mathcal{H}}$ and the Herbrand base $\mathcal{B}_{\mathcal{H}}$ are countably infinite, whereas $\mathcal{I}_{\mathcal{H}}$, the set of Herbrand interpretations for $\mathcal{B}_{\mathcal{H}}$, has as many elements as real numbers and the probability of each interpretation is infinitesimal or zero, numerically speaking. Also universally quantified formulas are likely to have probability zero when $\mathcal{U}_{\mathcal{H}}$ is infinite. ${ }^{14}$ Hence if $K B$ contains non-ground clauses, it is impossible in general, as $P_{c}(K B)=0$, to compute the conditional probability of a closed formula $\varphi$ by $P_{c}(\varphi \mid K B)=\frac{P_{c}(\varphi \wedge K B)}{P_{c}(K B)}$.

Nonetheless, $P_{c}(\varphi=y \mid K B=x)(x, y \in\{0,1\})$ is measure-theoretically definable as the Radon-Nikodym derivative (Feller 1971). The problem is that this measure-theoretic $P_{c}(\varphi=y \mid K B=x)$ is not unique at $K B=1$ because $P_{c}(K B=1)=0$. In other words, we have to choose, or have to construct an appropriate probability measure as $P_{c}(\cdot \mid K B=1)$, hopefully as an extension of the finite case. In particular we require $P_{c}(\varphi=1 \mid K B=1)=1$ hold if $K B \vdash \varphi$. In what follows, we construct such $P_{c}(\cdot \mid K B=1)$ by considering an infinite sequence of joint distributions $P_{c}\left(X_{1}=x_{1}, \ldots, X_{k}=x_{k} \mid \phi_{1}, \ldots, \phi_{n}\right)(n>0, k>0)$ and their limit, where $\phi_{1}, \ldots, \phi_{n}$ are ground clauses from $K B$. Before going into details, we look at an example to get a feeling for the infinite case.

Suppose $\mathcal{L}$ contains a unary predicate symbol $q / 1$, a constant symbol 0 and a unary function symbol $s / 1$. Then the Herbrand base $\mathcal{B}_{\mathcal{H}}$ is $\{q(0), q(s(0)), \ldots\}$. For the sake of brevity, we write $\overbrace{s(\cdots s(0) \cdots)}^{i} i$ as $i$ and the successor of $i(i \geq 0)$ as $i+1$. As stated in Sect. 2, a Herbrand interpretation for $\mathcal{B}_{\mathcal{H}}{ }^{\prime}$ is identified with an infinite $0-1$ vector $\left(x_{0}, x_{1}, \ldots\right)$ which specifies the truth value of $q(i)$ as $x_{i}\left(i \geq 0, x_{i} \in\{0,1\}\right)$. Let $P^{\prime}{ }_{c}(\cdot)$ be a product probability measure satisfying (by abuse of notation) $P^{\prime}{ }_{c}\left(q(0)=x_{0}, q(1)=x_{1}, \ldots\right)=\prod_{i=0}^{\infty} P^{\prime}{ }_{c}\left(q(i)=x_{i}\right)$ where $P^{\prime}{ }_{c}\left(q(i)=x_{i}\right)=1 / 2\left(i \geq 0, x_{i} \in\{0,1\}\right)$.

$$
\text { Put } \varphi \stackrel{\text { def }}{=} \forall x(q(x) \Rightarrow q(x+1)) \text { and define } \varphi_{n}(n \geq 0) \text { by }
$$

$$
\varphi_{n} \stackrel{\text { def }}{=} \forall x<n(q(x) \Rightarrow q(x+1))
$$

\footnotetext{
${ }^{14}$ If for example $P_{c}(q(n))<\alpha<1$ for every natural number $n, P_{c}(\forall x q(x))=\lim _{k} \alpha^{k}=0$.
} 


$$
=(q(0) \Rightarrow q(1)) \wedge \cdots \wedge(q(n-1) \Rightarrow q(n)) .
$$

We find, by calculation,

$$
\begin{aligned}
P_{c}^{\prime}\left(\varphi_{n}\right) & =\left(\frac{1}{2}\right)^{n+1}(n+2), \\
P_{c}^{\prime}{ }_{c}\left((q(0) \vee \cdots \vee q(k)) \wedge \varphi_{n}\right) & =\left(\frac{1}{2}\right)^{n+1}(k+1) \quad(n \geq k+1), \\
P^{\prime}{ }_{c}\left(\exists x q(x) \wedge \varphi_{n}\right) & =\left(\frac{1}{2}\right)^{n+1}(n+2) \quad\left(\text { because } P_{c}^{\prime}(\exists x q(x))=1\right) .
\end{aligned}
$$

Accordingly we have

$$
\begin{aligned}
P_{c}^{\prime}(\varphi) & =P_{c}^{\prime}(\forall x(q(x) \Rightarrow q(x+1))) \\
& =\lim _{n \rightarrow \infty} P^{\prime}{ }_{c}\left(\varphi_{n}\right) \\
& =\lim _{n \rightarrow \infty}\left(\frac{1}{2}\right)^{n+1}(n+2)=0, \\
P_{c}^{\prime}(q(0) \vee \cdots \vee q(k) \mid \varphi) & \stackrel{\text { def }}{=} \lim _{n \rightarrow \infty} P^{\prime}{ }_{c}\left(q(0) \vee \cdots \vee q(k) \mid \varphi_{n}\right) \\
& =\lim _{n \rightarrow \infty} \frac{k+1}{n+2}=0, \\
P^{\prime}{ }_{c}(\exists x q(x) \mid \varphi) & \stackrel{\text { def }}{=} \lim _{n \rightarrow \infty} P^{\prime}{ }_{c}\left(\exists x q(x) \mid \varphi_{n}\right) \\
& =1 .
\end{aligned}
$$

We note that although $P^{\prime}{ }_{c}(\varphi)=0$, conditional probabilities $P^{\prime}{ }_{c}(q(0) \vee \cdots \vee q(k) \mid \varphi)$ and $P^{\prime}{ }_{c}(\exists x q(x) \mid \varphi)$ have definite values, 0 and 1 respectively, if they are computed as limits of $P^{\prime}{ }_{c}\left(\cdot \mid \varphi_{n}\right)$ using successive approximations $\varphi_{n}$ to $\varphi$. However we also note that $1=P^{\prime}{ }_{c}(\exists x q(x) \mid \varphi) \neq \lim _{k \rightarrow \infty} P^{\prime}{ }_{c}(q(0) \vee \cdots \vee q(k) \mid \varphi)=0$. This means our limiting procedure which assigns probabilities to all formulas independently all at once does not yield a countably additive probability measure. So we next construct a conditional probability measure by repeating a limiting procedure and defining increasingly larger joint distributions to which Kolmogorov's extension theorem applies.

\subsection{Infinite domains}

Suppose $\mathcal{L}$ has function symbols. So the Herbrand universe $\mathcal{U}_{\mathcal{H}}$ and the Herbrand base $\mathcal{B}_{\mathcal{H}}$ are countably infinite. Let $A_{1}, A_{2}, \ldots$ be an enumeration of atoms in $\mathcal{B}_{\mathcal{H}}$. $\mathcal{I}_{\mathcal{H}}$, the set of all Herbrand interpretations for $\mathcal{B}_{\mathcal{H}}$, is written as $\mathcal{I}_{\mathcal{H}}=\prod_{i=0}^{\infty}\{0,1\}_{i}$ where $\{0,1\}_{i}$ represents the truth values of $A_{i}$. We consider each $\{0,1\}_{i}$ as a probability space with the discrete topology and give the product topology to $\mathcal{I}_{\mathcal{H}}$. We construct a probability space $\left(\mathcal{I}_{\mathcal{H}}, \mathcal{F}, P_{c}\right)$ where $P_{c}(\cdot)$ is an infinite product measure on $\mathcal{F}$ which is the smallest $\sigma$-algebra containing all open sets of $\mathcal{I}_{\mathcal{H}}$ (Feller 1971). $P_{c}\left(A_{1}=x_{1}, A_{2}=x_{2}, \ldots, A_{n}=x_{n}\right)=\prod_{i=1}^{n} P_{c}\left(A_{i}=x_{i}\right)$ holds for every $n>0$. Let $\phi_{1}, \phi_{2}, \ldots$ be an enumeration of ground clauses from $K B$. In the Herbrand universe, $K B$ is logically equivalent to $\bigwedge_{i>0} \phi_{i}$. 
Define an infinite sequence $\left(n_{0, i}\right)_{i>0}$ by

$$
n_{0, i}=i(i>0) .
$$

Now we are going to inductively construct a subsequence $\left(n_{k, i}\right)_{i>0}$ of $\left(n_{0, i}\right)_{i>0}$ for each $k$ $(k \geq 0)$ so that eventually $\left(n_{i, i}\right)_{i>0}$, a subsequence of every subsequence, makes a distribution sequence $P_{c}\left(A_{1}=x_{1}, \ldots, A_{k}=x_{k} \mid \phi_{1}, \ldots, \phi_{n_{i, i}}\right)(i=1,2, \ldots)$ convergent for all $k>0$ and the $x_{j}$ 's.

Suppose $\left(n_{k-1, i}\right)_{i>0}$, a subsequence of $\left(n_{0, i}\right)_{i>0}$, has been defined for $k(k>0)$ and $\lim _{i \rightarrow \infty} P_{c}\left(A_{1}=x_{1}, \ldots, A_{k-1}=x_{k-1} \mid \phi_{1}, \ldots, \phi_{n_{k-1, i}}\right)^{15}$ exists for every $x_{1}, \ldots, x_{k-1} \in$ $\{0,1\}$ if $k>1$. Choose a subsequence $\left(n_{k, i}\right)_{i>0}$ of $\left(n_{k-1, i}\right)_{i>0}$ such that

$$
P_{k, \infty}\left(A_{1}=x_{1}, \ldots, A_{k}=x_{k}\right) \stackrel{\text { def }}{=} \lim _{i \rightarrow \infty} P_{c}\left(A_{1}=x_{1}, \ldots, A_{k}=x_{k} \mid \phi_{1}, \ldots, \phi_{n_{k, i}}\right)
$$

exists for every $x_{1}, \ldots, x_{k} \in\{0,1\} .{ }^{16}$ Since $\left(n_{k, i}\right)_{i>0}$ is a subsequence of $\left(n_{k-1, i}\right)_{i>0}$, we have

$$
\begin{aligned}
& \sum_{x_{k}} \lim _{i \rightarrow \infty} P_{c}\left(A_{1}=x_{1}, \ldots, A_{k}=x_{k} \mid \phi_{1}, \ldots, \phi_{n_{k, i}}\right) \\
& \quad=\sum_{x_{k}} \lim _{i \rightarrow \infty} P_{c}\left(A_{1}=x_{1}, \ldots, A_{k}=x_{k} \mid \phi_{1}, \ldots, \phi_{n_{k-1, i}}\right) \\
& \quad=\lim _{i \rightarrow \infty} \sum_{x_{k}} P_{c}\left(A_{1}=x_{1}, \ldots, A_{k}=x_{k} \mid \phi_{1}, \ldots, \phi_{n_{k-1, i}}\right) \\
& =\lim _{i \rightarrow \infty} P_{c}\left(A_{1}=x_{1}, \ldots, A_{k-1}=x_{k-1} \mid \phi_{1}, \ldots, \phi_{n_{k-1, i}}\right) \\
& =P_{k-1, \infty}\left(A_{1}=x_{1}, \ldots, A_{k-1}=x_{k-1}\right) .
\end{aligned}
$$

Repeat this process and define $\left(n_{k-1, i}\right)_{i>0}$ for all $k(k>0)$. Then consider $\left(n_{i, i}\right)_{i>0} \cdot\left(n_{i, i}\right)_{i \geq k}$, the sequence with the initial $k-1$ elements removed from $\left(n_{i, i}\right)_{i>0}$, is a subsequence of $\left(n_{k, i}\right)_{i>0}$ for every $k(k>0)$. Therefore,

$$
\begin{aligned}
& \lim _{i \rightarrow \infty} P_{c}\left(A_{1}=x_{1}, \ldots, A_{k}=x_{k} \mid \phi_{1}, \ldots, \phi_{n_{i, i}}\right) \\
& \quad=\lim _{i \rightarrow \infty} P_{c}\left(A_{1}=x_{1}, \ldots, A_{k}=x_{k} \mid \phi_{1}, \ldots, \phi_{n_{k, i}}\right) \\
& \quad=P_{k, \infty}\left(A_{1}=x_{1}, \ldots, A_{k}=x_{k}\right)
\end{aligned}
$$

holds for every $k>0$ and $x_{1}, \ldots, x_{k} \in\{0,1\}$. Also by construction, it holds that

$$
\begin{aligned}
& \sum_{x_{k}} P_{k, \infty}\left(A_{1}=x_{1}, \ldots, A_{k}=x_{k}\right) \\
& \quad=\sum_{x_{k}} \lim _{i \rightarrow \infty} P_{c}\left(A_{1}=x_{1}, \ldots, A_{k}=x_{k} \mid \phi_{1}, \ldots, \phi_{n_{k, i}}\right)
\end{aligned}
$$

\footnotetext{
${ }^{15}$ In this section, a formula $\phi$ on the conditioning part always means $\phi=1$. That is, $P_{c}(\cdot \mid \phi)$ means $P_{c}(\cdot \mid$ $\phi=1)$.

${ }^{16}$ We can always choose such a convergent subsequence as there are only finitely many $\left(x_{1}, \ldots, x_{k-1}\right)$ s and a countably infinite set $\left\{P_{c}\left(A_{1}=x_{1}, \ldots, A_{k-1}=x_{k-1} \mid \phi_{1}, \ldots, \phi_{n_{k-1, i}}\right) \mid x_{j} \in\{1,0\}, i>0\right\}$ has a cluster point in $[0,1]$.
} 


$$
=P_{k-1, \infty}\left(A_{1}=x_{1}, \ldots, A_{k-1}=x_{k-1}\right) \text { by (3). }
$$

So we can apply Kolmogorov's extension theorem (Chow and Teicher 1997) to the set of "consistent" joint distributions $\left\{P_{k, \infty}\left(A_{1}=x_{1}, \ldots, A_{k}=x_{k}\right) \mid k>0\right\}$ and conclude that a probability measure denoted by $P_{c}^{\infty}\left(\cdot \mid \bigwedge_{i>0} \phi_{i}\right)$ on $\mathcal{I}_{\mathcal{H}}$ having $P_{k, \infty}\left(A_{1}=x_{1}, \ldots, A_{k}=\right.$ $\left.x_{k}\right)$ s as marginal distributions exists:

$$
\begin{aligned}
P_{c}^{\infty} & \left(A_{1}=x_{1}, \ldots, A_{k}=x_{k} \mid \bigwedge_{i>0} \phi_{i}\right) \\
& =P_{k, \infty}\left(A_{1}=x_{1}, \ldots, A_{k}=x_{k}\right) \\
& =\lim _{i \rightarrow \infty} P_{c}\left(A_{1}=x_{1}, \ldots, A_{k}=x_{k} \mid \phi_{1}, \ldots, \phi_{n_{i, i}}\right) \text { by (4). }
\end{aligned}
$$

We summarize the argument so far as

Theorem 4 Suppose $K B$ is a set of countably many clauses in a first-order language $\mathcal{L}$ which may contain function symbols. Let $\mathcal{I}_{\mathcal{H}}$ be the Herbrand interpretations for $\mathcal{L}$ and $P_{c}(\cdot)$ a probability measure on $\mathcal{I}_{\mathcal{H}}$ such that $P_{c}\left(A_{1}=x_{1}, \ldots, A_{k}=x_{k}\right)=\prod_{i=1}^{k} P_{c}\left(A_{i}=x_{i}\right)\left(x_{i} \in\right.$ $\{0,1\})$ for every $k(k>0)$ where $A_{1}, A_{2}, \ldots$ is an enumeration of ground atoms in $\mathcal{L}$. Let $\phi_{1}, \phi_{2}, \ldots$ be an enumeration of ground clauses from $K B$. We assume $P_{c}\left(\phi_{1} \wedge \cdots \wedge \phi_{m}\right)>0$ for every $m>0 .{ }^{17}$

Then there exist an infinite sequence $\left(n_{i, i}\right)_{i>0}$ and a probability measure on $\mathcal{I}_{\mathcal{H}}$ denoted by $P_{c}^{\infty}\left(\cdot \mid \bigwedge_{i>0} \phi_{i}\right)$ or equivalently by $P_{c}^{\infty}(\cdot \mid K B)$ such that $\lim _{i \rightarrow \infty} P_{c}\left(A_{1}=x_{1}, \ldots, A_{k}=\right.$ $\left.x_{k} \mid \phi_{1}, \ldots, \phi_{n_{i, i}}\right)$ converges to a joint distribution $P_{c}^{\infty}\left(A_{1}=x_{1}, \ldots, A_{k}=x_{k} \mid K B\right)$ for every $k>0$. Moreover, if $K B \vdash \phi$ holds for an existentially closed formula $\phi,{ }^{18}$ we have $P_{c}^{\infty}(\phi \mid$ $K B)=1$.

Proof We have only to prove the latter part. Suppose $K B \vdash \phi$ and $\phi$ is existentially closed. We consider the universally quantified formula $\neg \phi$ as a set of clauses.

Since $K B \cup\{\neg \phi\}$ is inconsistent, it follows from Herbrand's theorem that there are ground clauses $\left\{\phi_{1}, \ldots, \phi_{N}\right\}$ from $K B$ and those $\left\{\psi_{1}, \ldots, \psi_{M}\right\}$ from $\neg \phi$ such that $\phi_{1}, \ldots, \phi_{N} \vdash \neg\left(\psi_{1} \wedge \cdots \wedge \psi_{M}\right)$. So $\phi_{1}, \ldots, \phi_{n_{i, i}} \vdash \neg\left(\psi_{1} \wedge \cdots \wedge \psi_{M}\right)$ holds for every $i$ such that $\left\{\phi_{1}, \ldots, \phi_{n_{i, i}}\right\} \supseteq\left\{\phi_{1}, \ldots, \phi_{N}\right\}$, and hence we have $P_{c}\left(\neg\left(\psi_{1} \wedge \cdots \wedge \psi_{M}\right) \mid\right.$ $\left.\phi_{1}, \ldots, \phi_{n_{i, i}}\right)=1$ for large $i$. Therefore, by taking the limit, we conclude $P_{c}^{\infty}\left(\neg\left(\psi_{1} \wedge \cdots \wedge\right.\right.$ $\left.\left.\psi_{M}\right) \mid K B\right)=1$. On the other hand, since $\neg \phi \cup\left\{\neg\left(\psi_{1} \wedge \cdots \wedge \psi_{N}\right)\right\}$ has no Herbrand model, we have $\neg\left(\psi_{1} \wedge \cdots \wedge \psi_{N}\right) \vdash \phi$. It follows from $P_{c}^{\infty}\left(\neg\left(\psi_{1} \wedge \cdots \wedge \psi_{M}\right) \mid K B\right)=1$ that $P_{c}^{\infty}(\phi \mid K B)=1$ as well.

We remark that $P_{c}^{\infty}(\cdot \mid K B)$ depends on $\left(n_{i, i}\right)_{i>0}$ and may not be unique. A simple uniqueness condition is as follows. Let $\phi_{1}, \phi_{2}, \ldots$ be an enumeration of ground clauses from $K B$. Suppose $P_{c}(K B)=\lim _{i \rightarrow \infty} P_{c}\left(\phi_{1} \wedge \cdots \wedge \phi_{i}\right)>0$ exists. ${ }^{19}$ Then $P_{c}^{\infty}(\cdot \mid K B)$ is unique and coincides with $P_{c}(\cdot \mid K B)$. This is because $P_{c}(\varphi \mid K B)=\lim _{i \rightarrow \infty} P_{c}\left(\varphi \mid \phi_{1} \wedge \cdots \wedge \phi_{i}\right)=$ $\lim _{i \rightarrow \infty} P_{c}\left(\varphi \mid \phi_{1} \wedge \cdots \wedge \phi_{n_{i, i}}\right)=P_{c}^{\infty}(\varphi \mid K B)^{20}$ holds for any Boolean formula $\varphi$. When

\footnotetext{
${ }^{17}$ This is satisfied if $0<P_{c}\left(A_{i}\right)<1$ for all $i(i>0)$.

${ }^{18} \mathrm{~A}$ formula is existentially closed if it is closed and takes the form $\exists x_{1}, \ldots, x_{n} \varphi$ where $\varphi$ has no quantifier.

${ }^{19}$ Apparently the limit does not depend on the choice of enumeration.

${ }^{20}$ Any convergent subsequence of a convergent sequence has the same limit.
} 
$P_{c}(K B)=0$ however, the uniqueness condition is still an open question. We need to check the uniqueness of $P_{c}^{\infty}(\cdot \mid K B)$ for each case at the moment.

Unlike Proposition 1, the inverse of Theorem 4, i.e. $P_{c}^{\infty}(\phi \mid K B)=1$ implies $K B \vdash$ $\phi$, does not necessarily hold in infinite domains. Here is a counter example. Let $\varphi=$ $\forall x(q(x) \Rightarrow q(x+1)), \varphi_{n}=(q(0) \Rightarrow q(1)) \wedge \cdots \wedge(q(n-1) \Rightarrow q(n))$ and $P^{\prime}{ }_{c}(\cdot)$ be as in the previous subsection. We see that $\lim _{n \rightarrow \infty} P^{\prime}{ }_{c}\left(q(0)=x_{0}, \ldots, q(k)=x_{k} \mid \varphi_{n}\right)$ always converges for any $x_{0}, \ldots, x_{k}$ and the resulting $P_{c}^{\prime \infty}(\cdot \mid \varphi)$ is unique regardless of the choice of $\left(n_{i, i}\right)_{i>0}$. Thus $\left(\varphi_{n}\right)_{n \geq 0}$ defines an infinite CBPM $P_{c}^{\prime \infty}(\cdot \mid \varphi)$. We have $P_{c}^{\prime \infty}(\neg q(0) \mid \varphi)=1$ because $P_{c}^{\prime \infty}(q(0) \mid \varphi)=\lim _{n \rightarrow \infty} P^{\prime}{ }_{c}\left(q(0) \mid \varphi_{n}\right)=0$. We on the other hand have $\varphi \nvdash \neg q(0)$ because $\varphi$ has a Herbrand model that makes every ground atom true. So $P_{c}^{\prime \infty}(\neg q(0) \mid \varphi)=1$ but not $\varphi \nvdash \neg q(0)$.

From the next section on, we get back to finite domains and consider probability computation and parameter learning in finite domains.

\section{Parameter learning}

In this section we assume $K B$ is a Boolean formula (finite set of ground clauses) and derive the EMC algorithm, an EM algorithm for CBPMs which statistically estimates parameters from data. It is a descendent of the FAM algorithm for SLPs (Cussens 2001) such that conditioning by a "success" proposition in FAM is generalized to conditioning by a knowledge base $K B$.

\subsection{Probability computation by BDDs}

Before deriving the EMC algorithm, we describe how probabilities of probabilistic Boolean formulas are computed by BDDs (binary decision diagrams) (Akers 1978; Bryant 1986). ${ }^{21}$ The description is sketchy due to space limitations.

Suppose a probabilistic Boolean formula $\varphi$ consisting of independent Boolean variables is given. To compute $P_{c}(\varphi)$, we convert $\varphi$ to a BDD which is a directed acyclic graph representing a disjunctive normal form of $\varphi$ such that disjuncts are mutually exclusive. In the graph, each non-terminal node $N$ is labeled by a Boolean variable, $A$, and has two outgoing edges to its children, 1 -edge and 0 -edge, respectively representing the assignments $A=$ true and $A=$ false. There are two types of terminal nodes, 1 -terminal and 0 -terminal. Each path from the root node to a 1-terminal represents an assignment for the Boolean variables in $\varphi$ making $\varphi$ true, thereby corresponding to a disjunct in a disjunctive normal form $\bigvee_{i=1}^{n}\left(L_{i, 1} \wedge \cdots \wedge L_{i, k_{i}}\right)$ for $\varphi$. Let $\operatorname{BDD}_{\varphi}$ be a $\operatorname{BDD}$ for $\varphi . \mathrm{BDD}_{\varphi}$ is unique as a graph once a variable ordering in $\varphi$ is fixed. The size of $\mathrm{BDD}_{\varphi}$ heavily depends on the variable ordering and finding the best ordering is NP-hard.

$P_{c}(\varphi)$ is naively computed as

$$
P_{c}\left(\bigvee_{i=1}^{n} \bigwedge_{j=1}^{k_{i}} L_{i, j}\right)=\sum_{i=1}^{n} \prod_{j=1}^{k_{i}} P_{c}\left(L_{i, j}\right)
$$

\footnotetext{
${ }^{21}$ Similar probability computation is possible by ZDDs (zero-suppressed BDDs) (Minato 2001). For the full details of probability computation by BDDs and ZDDs, see (Ishihata et al. 2008), downloadable at http://www.cs.titech.ac.jp/ tr/reports/2008/TR08-0004.pdf.
} 
where for a literal $L(L=A$ or $L=\neg A$ for some atom $A), P_{c}(L)=P_{c}(A)$ if $L=A$ else $1-P_{c}(A)$. It is possible however to perform the same computation much more efficiently based on $\mathrm{BDD}_{\varphi}$ by taking advantage of the fact that subgraphs in $\mathrm{BDD}_{\varphi}$ are shared and hence probability computations for subgraphs can also be shared. So we compute backward probabilities for all nodes in $\mathrm{BDD}_{\varphi}$ by dynamic programming, starting from terminal nodes then upward in which the backward probability for a non-terminal node is computed as the sum of those for its child nodes. $P_{c}(\varphi)$ is obtained as the backward probability for the root node. It is computed in time proportional to the number of edges in $\operatorname{BDD}_{\varphi}$.

Applying the BDD probability computation to parameter estimation by the EM algorithm (Dempster et al. 1977) is more complicated. The backward probability computation described above is just one of the four types of probability computation done in the BDD-EM algorithm which is a BDD-based EM algorithm for probabilistic Boolean formulas (Ishihata et al. 2008; Inoue et al. 2009). It uses "decomposed BDDs", hierarchically organized BDDs, and computes by dynamic programming forward probability, inside-probability and outside-probability in addition to backward probability. Using BDDs is not the only one choice though. We may use ZDDs instead of BDDs. Then for example, parameter learning for PCFGs is done in $O\left(N^{3}\right)$ per iteration by the ZDD-EM algorithm where $N$ is the sentence length (Ishihata et al. 2008), exactly the same order as the standard Inside-Outside algorithm for PCFGs. Although details are skipped, the EMC algorithm introduced next employs the same computation techniques as the $\mathrm{BDD}(\mathrm{ZDD})$-EM algorithm to compute required probabilities and expectations.

\subsection{Deriving the EMC algorithm}

Suppose we have a CBPM $P_{c}(\cdot \mid K B, \boldsymbol{\theta})$ and let $O_{1}, \ldots, O_{T}(T>0)$ be Boolean formulas representing i.i.d. observations. We estimate parameters $\boldsymbol{\theta}$, i.e. probabilities of atoms being true, from $O_{1}, \ldots, O_{T}$ by MLE (maximum likelihood estimation) using the following likelihood function.

$$
\mathbf{L}(\boldsymbol{\theta})=\prod_{t=1}^{T} P_{c}\left(O_{t} \mid K B, \boldsymbol{\theta}\right) .
$$

Since atoms appearing in $K B$ other than those in $O_{1}, \ldots, O_{T}$ are not observed, we use the EM algorithm (Dempster et al. 1977) to find $\boldsymbol{\theta}$ that maximizes $\mathbf{L}(\boldsymbol{\theta})$. Let $x$ be a Herbrand interpretation considered as a $0-1$ vector as before and $P_{c}(x \mid \boldsymbol{\theta})$ the underlying joint distribution that makes atoms in $\mathcal{B}_{\mathcal{H}}$ independent.

To deal with the existence of i.i.d. atoms in probabilistic models, we assume $\mathcal{B}_{\mathcal{H}}$ is partitioned into a set $\mathcal{C}$ of sets $s$ of i.i.d. atoms. Every atom $A \in s(s \in \mathcal{C})$ has the same parameters $\theta_{s, 1}=P_{c}(A)$ and $\theta_{s, 0}=P_{c}(\neg A)$. We use $\sigma_{s, v}(x)(s \in \mathcal{C}, v \in\{1,0\})$ for the count of atoms in $s$ that take on $v$ in the Herbrand interpretation $x$. We can write $\boldsymbol{\theta}=\left\{\theta_{s, 1}, \theta_{s, 0} \mid s \in \mathcal{C}\right\}$.

Let $P_{c}\left(x_{t}, O_{t} \mid K B, \boldsymbol{\theta}\right)$ be a conditional joint distribution over Herbrand interpretations $x_{t}$ and the $t$-th observation $O_{t}$ whose marginal distribution is $P_{c}\left(O_{t} \mid K B, \boldsymbol{\theta}\right)$. Since it is not just a joint distribution but a conditional one, we follow (Cussens 2001) and derive the $Q$ function as follows. First introduce a new joint distribution $\widetilde{P}(\cdot)$ below:

$$
\begin{aligned}
& \widetilde{P}\left(\left\langle u^{(1)}, \ldots, u^{(k-1)}\right\rangle, x_{t}, O_{t} \mid K B, \boldsymbol{\theta}\right) \\
& \stackrel{\text { def }}{=} P_{c}\left(u^{(1)}, \neg K B \mid \boldsymbol{\theta}\right) \cdots P_{c}\left(u^{(k-1)}, \neg K B \mid \boldsymbol{\theta}\right) P_{c}\left(x_{t}, O_{t}, K B \mid \boldsymbol{\theta}\right)
\end{aligned}
$$


Step 1: Initialize parameters $\boldsymbol{\theta}^{(0)}$

Step 2: Iterate $\boldsymbol{\theta}^{(k)}=\operatorname{argmax}_{\boldsymbol{\theta}} Q\left(\boldsymbol{\theta}, \boldsymbol{\theta}^{(k-1)}\right)$ until convergence of $\mathbf{L}\left(\boldsymbol{\theta}^{(k)}\right)$

Step 3: Return $\boldsymbol{\theta}^{(\infty)}=\boldsymbol{\theta}^{(k)}$ at convergence as estimated values

Fig. 1 The abstract EM algorithm

where the $u^{(j)}$ 's are independent Herbrand interpretations. It holds that

$$
\begin{aligned}
& \sum_{k=1}^{\infty} \sum_{\left\langle u^{(1)}, \ldots, u^{(k-1)}\right\rangle} \widetilde{P}\left(\left\langle u^{(1)}, \ldots, u^{(k-1)}\right\rangle, x_{t}, O_{t} \mid K B, \boldsymbol{\theta}\right) \\
& \quad=\sum_{k=1}^{\infty}\left(\sum_{u} P_{c}(u, \neg K B \mid \boldsymbol{\theta})\right)^{k-1} P_{c}\left(x_{t}, O_{t}, K B \mid \boldsymbol{\theta}\right)=P_{c}\left(x_{t}, O_{t} \mid K B, \boldsymbol{\theta}\right) .
\end{aligned}
$$

Accordingly $P_{c}\left(O_{t} \mid K B, \boldsymbol{\theta}\right)$ is a marginal distribution of $\widetilde{P}\left(\left\langle u^{(1)}, \ldots, u^{(k-1)}\right\rangle, x_{t}, O_{t} \mid\right.$ $K B, \boldsymbol{\theta})$. Hence putting $\bar{u}_{t}^{k-1}=\left\langle u^{(1)}, \ldots, u^{(k-1)}\right\rangle$ and assuming $\bar{u}_{t}^{k-1}, x_{t}$ as hidden variables in $\widetilde{P}\left(\bar{u}_{t}^{k-1}, x_{t}, O_{t} \mid K B, \boldsymbol{\theta}^{\prime}\right)$, we introduce $Q\left(\boldsymbol{\theta}, \boldsymbol{\theta}^{\prime}\right)$ by

$$
\begin{aligned}
Q\left(\boldsymbol{\theta}, \boldsymbol{\theta}^{\prime}\right) \stackrel{\text { def }}{=} & \sum_{t=1}^{T} \sum_{k=1}^{\infty} \sum_{x_{t}} \sum_{\bar{u}_{t}^{k-1}} \widetilde{P}\left(\bar{u}_{t}^{k-1}, x_{t} \mid O_{t}, K B, \boldsymbol{\theta}^{\prime}\right) \ln \widetilde{P}\left(\bar{u}_{t}^{k-1}, x_{t}, O_{t} \mid K B, \boldsymbol{\theta}\right) \\
= & \frac{T}{P_{c}\left(K B \mid \boldsymbol{\theta}^{\prime}\right)}\left(\sum_{u: u \models \neg K B} P_{c}\left(u \mid \boldsymbol{\theta}^{\prime}\right) \ln P_{c}(u \mid \boldsymbol{\theta})\right) \\
& +\sum_{t=1}^{T} \frac{1}{P_{c}\left(O_{t} \wedge K B \mid \boldsymbol{\theta}^{\prime}\right)} \sum_{x_{t}: x_{t} \models O_{t} \wedge K B} P_{c}\left(x_{t} \mid \boldsymbol{\theta}^{\prime}\right) \ln P_{c}\left(x_{t} \mid \boldsymbol{\theta}\right) .
\end{aligned}
$$

Using this $Q\left(\boldsymbol{\theta}, \boldsymbol{\theta}^{\prime}\right)$, the EM algorithm is abstractly described in Fig. 1.

Since $Q\left(\boldsymbol{\theta}, \boldsymbol{\theta}^{\prime}\right) \geq Q\left(\boldsymbol{\theta}^{\prime}, \boldsymbol{\theta}^{\prime}\right) \Rightarrow \mathbf{L}(\boldsymbol{\theta}) \geq \mathbf{L}\left(\boldsymbol{\theta}^{\prime}\right)$ is provable, $\mathbf{L}\left(\boldsymbol{\theta}^{(k)}\right)$ is guaranteed to increase at every iteration. Note that $\boldsymbol{\theta}^{(\infty)}$ only gives a local maximum of $\mathbf{L}(\boldsymbol{\theta})$, not necessarily the global maximum. By substituting (6) for $Q\left(\boldsymbol{\theta}, \boldsymbol{\theta}^{\prime}\right)$ in Fig. 1, we obtain the EMC algorithm (EM algorithm for constraint-based probabilistic models) in Fig. 2.

As explained previously, we adopted BDD(ZDD)-based probability computation when we implemented the EMC algorithm. We call the resulting algorithm the BDD-EMC algorithm. Compared to the BDD-EM algorithm (Ishihata et al. 2008) it additionally computes the first term in (7) which is the average number of i.i.d. atoms in $s$ that take on the value $v$ in a Herbrand interpretation falsifying $K B$. Although $\mathrm{BDD}(\mathrm{ZDD})$-based probability computation techniques may help us but computing $P_{c}(K B \mid \theta)$ is still infeasible when $K B$ is large. It remains as a future research topic for CBPMs.

\section{Constraint-based statistical abduction}

In this section we apply CBPMs to statistical abduction. We assume domains are finite. 
Put $\left\{\begin{array}{l}\mathcal{W}_{1}=\{u \mid u \models \neg K B\} \\ \mathcal{W}_{2}^{t}=\left\{x_{t} \mid x_{t} \models O_{t} \wedge K B\right\} \quad(1 \leq t \leq T)\end{array}\right.$

where $\mathcal{W}_{1}$ (resp. $\mathcal{W}_{2}^{t}$ ) is the set of truth assignments for $\mathcal{B}_{\mathcal{H}}$ which make $K B$ false (resp. $O_{t} \wedge K B$ true), and repeat the $\mathbf{E}$-step and the M-step below alternately until convergence.

E-step: Compute the conditional expectation $\eta_{\theta}^{v}[s]$ of $\sigma_{s, v}$ for $s \in \mathcal{C}, v \in\{0,1\}$ by

$$
\begin{aligned}
\eta_{\theta}^{v}[s]= & \frac{T}{P_{c}(K B)} \sum_{u \in \mathcal{W}_{1}} \sigma_{s, v}(u) \prod_{s^{\prime} \in \mathcal{C}} \prod_{v^{\prime} \in\{1,0\}} \theta_{s^{\prime}, v^{\prime}}^{\sigma_{s^{\prime}, v^{\prime}}(u)} \\
& +\sum_{t=1}^{T} \frac{1}{P_{c}\left(O_{t} \wedge K B\right)} \sum_{x_{t} \in \mathcal{W}_{2}^{t}} \sigma_{s, v}\left(x_{t}\right) \prod_{s^{\prime} \in \mathcal{C}} \prod_{v^{\prime} \in\{1,0\}} \theta_{s^{\prime}, v^{\prime}}^{\sigma_{s^{\prime}, v^{\prime}}\left(x_{t}\right)}
\end{aligned}
$$

where

$$
\begin{aligned}
P_{c}(\neg K B \mid \boldsymbol{\theta}) & =\sum_{u \in \mathcal{W}_{1}} \prod_{s \in \mathcal{C}} \prod_{v \in\{1,0\}} \theta_{s, v}^{\sigma_{s, v}(u)}, \\
P_{c}(K B \mid \boldsymbol{\theta}) & =1-P_{c}(\neg K B \mid \boldsymbol{\theta}), \\
P_{c}\left(O_{t} \wedge K B \mid \boldsymbol{\theta}\right) & =\sum_{x_{t} \in \mathcal{W}_{2}^{t}} \prod_{s \in \mathcal{C}} \prod_{v \in\{1,0\}} \theta_{s, v}^{\sigma_{s, v}\left(x_{t}\right)} .
\end{aligned}
$$

M-step: Update $\boldsymbol{\theta}$ to $\hat{\boldsymbol{\theta}}$ by

$$
\hat{\theta}_{s, v}=\frac{\eta_{\theta}^{v}[s]}{\eta_{\theta}^{1}[s]+\eta_{\theta}^{0}[s]} \quad \text { for every } s \in \mathcal{C}, v \in\{0,1\}
$$

Fig. 2 The EMC algorithm

\subsection{Statistical abduction}

Abduction is one of three forms of logical inference (deduction, induction, abduction) that infers the best explanation $E$ for an observation $O$ such that $K B \wedge E \vdash O$ and $K B \wedge E$ is consistent. Statistical abduction in addition attempts to quantify explanations with probabilities and select the best explanation as the one having the highest probability, realizing robust abduction applicable to noisy data. The framework of statistical abduction is general. Many known probabilistic models from BNs to PCFGs are understood as performing statistical abduction (Sato and Kameya 2001). There are already a couple of systems for statistical abduction (Poole 1993, 1997; Sato and Kameya 2002). They are common in that $O$ is an atom representing our observation, $E$ is a conjunction made up of particular (probabilistic) atoms called abducibles, and $K B$ is a logic program of one kind or another that describes the process of how $O$ is deduced from $K B \wedge E$. One problem with these systems is that to ensure this procedural nature, $K B$ is restricted to definite clause programs (Poole 1993; 
Sato and Kameya 2002) or to acyclic logic programs (Poole 1997) ${ }^{22}$ that prevents the use of disjunctions and cyclic rules. Observations restricted to atoms is another problem. We may observe negative results $\neg$ happy (Bill) or complex events such as rich $($ Bill $) \Rightarrow$ happy $($ Bill $)$. Although these restrictions simplify inference and probability computation, they are unnecessarily restrictive from the viewpoint of knowledge representation.

To solve these problems, we propose constraint-based statistical abduction which applies CBPMs to statistical abduction. In the constraint-based statistical abduction, we have a knowledge base $K B$ which is a set of arbitrary clauses, not restricted to Horn clauses, and i.i.d. observations $O_{1}, \ldots, O_{T}$ which we assume to be arbitrary Boolean formulas made up of ground atoms. For each $O_{t}(1 \leq t \leq T)$, we search for an explanation $E$ in the search space $\mathcal{E}$ of possible explanations such that $K B \wedge E \vdash O_{t}$ and $K B \wedge E$ is consistent. $\mathcal{E}$ is specified beforehand, for instance as a set of conjunctions of abducibles as in PRISM. Let $\left\{E_{1}^{(t)}, \ldots, E_{k_{t}}^{(t)}\right\}$ be a set of explanations we obtain for $O_{t} \cdot{ }^{23}$ The disjunction $E^{(t)}=E_{1}^{(t)} \vee \cdots \vee E_{k_{t}}^{(t)}$ is called a disjunctive explanation for $O_{t}$ following PRISM. We then construct a CBPM $P_{c}(\cdot \mid K B, \theta)$ that specifies a distribution over Herbrand interpretations. Here $\boldsymbol{\theta}$ collectively stands for parameters, i.e. the probabilities of atoms in $\mathcal{B}_{\mathcal{H}}$ being true. We estimate $\boldsymbol{\theta}$ as the maximizer of the following likelihood function $\mathbf{L}^{a b d}(\boldsymbol{\theta})$.

$$
\mathbf{L}^{a b d}(\boldsymbol{\theta})=\prod_{t=1}^{T} P_{c}\left(E^{(t)} \mid K B, \boldsymbol{\theta}\right) .
$$

The reason for the choice of this likelihood function is as follows. First as a probabilistic model applied to i.i.d. data $O_{1}, \ldots, O_{T}$, we should maximize $\mathbf{L}(\boldsymbol{\theta})=\prod_{t=1}^{T} P_{c}\left(O_{t} \mid K B, \boldsymbol{\theta}\right)$. On the other hand as we are seeking for true explanations for the $O_{t}$ 's in statistical abduction, the probability of (at least) one of the $O_{t}$ 's explanations $\left\{E_{1}^{(t)}, \ldots, E_{k_{t}}^{(t)}\right\}$, or equivalently their disjunction $E^{(t)}$ being true, should be high. In other words, we should maximize $\mathbf{L}^{a b d}(\boldsymbol{\theta})=$ $\prod_{t=1}^{T} P_{c}\left(E^{(t)} \mid K B, \boldsymbol{\theta}\right)$ as well. Unfortunately parameters that maximize $\mathbf{L}^{a b d}(\boldsymbol{\theta})$ may differ from those that maximize $\mathbf{L}(\boldsymbol{\theta})$. We therefore maximize $\prod_{t=1}^{T} P_{c}\left(O_{t} \wedge E^{(t)} \mid K B, \boldsymbol{\theta}\right)$ as a compromise between the two. However in view of $K B \models E^{(t)} \Rightarrow O_{t}, P_{c}\left(O_{t} \wedge E^{(t)} \mid K B, \boldsymbol{\theta}\right)$ is equal to $P_{c}\left(E^{(t)} \mid K B, \boldsymbol{\theta}\right)$, thus reaching $\mathbf{L}^{a b d}(\boldsymbol{\theta})$ in (7). Moreover the EMC algorithm in Fig. 2 works for any $O_{t}$ s as long as they are Boolean formulas. So we can use it simply by replacing $O_{t}$ with $E^{(t)}$ in Fig. 2 to maximize $\mathbf{L}^{a b d}(\boldsymbol{\theta})$.

\subsection{Learning example}

We present here a small learning example for the understanding of constraint-based statistical abduction. It is often observed that smart people are rich and rich people are friends with rich people who are generous. The following $K B_{\text {rich }}$ formalizes this observation (free variables are implicitly universally quantified).

$$
K B_{\text {rich }}=\left\{\begin{array}{l}
\text { friend }(a, b), \\
\text { friend }(b, c), \\
\operatorname{generous}(b), \\
\operatorname{friend}(x, y) \Leftarrow \operatorname{friend}(y, x), \\
\operatorname{rich}(x) \Leftrightarrow \operatorname{smart}(x) \vee \exists y(\operatorname{friend}(x, y) \wedge \operatorname{rich}(y) \wedge \operatorname{generous}(y)) .
\end{array}\right.
$$

${ }^{22}$ Later the condition is relaxed to "contingently acyclic logic programs" (Poole 2000).

${ }^{23}$ Note that there may be infinitely many explanations but we assume they are finite. 
Table 1 Learned probabilities $P_{r}(F)$ for ground formulas $F$

\begin{tabular}{llll}
\hline$F$ & \multicolumn{2}{l}{ Observations } & \\
\cline { 2 - 3 } & $a[30 / 0] c[30 / 0]$ & $a[20 / 10] c[10 / 20]$ & 1.00000 \\
\hline friend $(a, b)$ & 1.00000 & 1.00000 & 1.00000 \\
friend $(b, c)$ & 1.00000 & 1.00000 & 0.49984 \\
friend $(c, a)$ & 0.42478 & 0.51797 & 0.45345 \\
generous $(a)$ & 0.68836 & 0.28644 & 1.00000 \\
generous $(b)$ & 1.00000 & 1.00000 & 0.57080 \\
generous $(c)$ & 0.89564 & 0.15686 & 0.00000 \\
$\operatorname{smart}(a)$ & 0.59494 & 0.58865 & 0.00000 \\
$\operatorname{smart}(b)$ & 1.00000 & 0.03550 & 0.00000 \\
$\operatorname{smart}(c)$ & 0.24699 & 0.12255 & 0.00000 \\
$\operatorname{rich}(a)$ & 1.00000 & 0.66666 & 0.00000 \\
$\operatorname{rich}(b)$ & 1.00000 & 0.28147 & 0.00000 \\
$\operatorname{rich}(c)$ & 1.00000 & 0.33334 & 0.00000 \\
$\operatorname{rich}(a) \wedge \neg$ rich $(b)$ & 0.00000 & 0.38520 &
\end{tabular}

The above $K B_{\text {rich }}$ is non-Horn. It says that there live three people $a, b$ and $c$ in the world where $a$ and $b$ are friends and so are $b$ and $c$ (but it is unknown whether or not $a$ and $c$ are friends). $b$ is known to be generous. We are sure that if $y$ is a friend of $x$, symmetrically, $x$ is a friend of $y$. Also it holds that $x$ is rich if-and-only-if $x$ is smart or has a friend who is rich and generous. Friendship is cyclic and being rich is also cyclic.

Suppose we have observed the state of $a$ and $c$ several times. If we observe $\operatorname{rich}(a)$ $n$ times while $\neg$ rich $(a) m$ times, we denote the observations by $a[n / m]$. Similarly for $c\left[n^{\prime} / m^{\prime}\right]$. We estimate the probability of $\operatorname{rich}(b)$ from observations $a[n / m]$ and $c\left[n^{\prime} / m^{\prime}\right]$. As the set of possible explanations for $\operatorname{rich}(a)$ for example, we take $\{\operatorname{smart}(a) \vee(\operatorname{friend}(a, y) \wedge$ $\operatorname{rich}(y) \wedge$ generous $(y)) \mid y \in\{a, b, c\}\}$, i.e. ground instantiations of the r.h.s. of the equivalence formula for $\operatorname{rich}(x)$, and dually the negation of the r.h.s. as the ones for $\neg \operatorname{rich}(a)$. Similarly for $\operatorname{rich}(c)$ and $\neg$ rich $(c)$. Under this abductive setting, we learned parameters $\boldsymbol{\theta}$ in $P_{c}\left(\cdot \mid K B_{r i c h}, \boldsymbol{\theta}\right)$ from observations $a[n / m]$ and $c\left[n^{\prime} / m^{\prime}\right]$ by the EMC algorithm varying $n$, $m, n^{\prime}$ and $m^{\prime}$.

Table 1 summarizes probabilities $P_{r}(F) \stackrel{\text { def }}{=} P_{c}\left(F \mid K B_{r i c h}, \boldsymbol{\theta}\right)$ specified by the learned $\boldsymbol{\theta}$ for various ground formulas $F$.

Columns correspond to each observation set. So for example $\operatorname{friend}(c, a)$ is true with probability 0.51797 when parameters are learned from observations $a[20 / 10] c[10 / 20]$, i.e. rich $(a)$ observed 20 times and $\neg$ rich $(a) 10$ times etc. ${ }^{24}$

In the table, facts in $K B_{\text {rich }}$ such as friend $(a, b)$ and generous $(b)$ receive probability one. Also we can confirm $\operatorname{rich}(a) \Leftarrow \operatorname{rich}(b)$, a logical consequence of $K B_{\text {rich }}$, has probability one by computing $P_{r}(\operatorname{rich}(a) \Leftarrow \operatorname{rich}(b))=P_{r}(\operatorname{rich}(a))+P_{r}(\neg \operatorname{rich}(b))-P_{r}(\operatorname{rich}(a) \wedge$ $\neg$ rich $(b))$ from the table. Learned probabilities seem to support our intuition that the chance of being rich is affected by friends. For example look at $b$. When both $a$ and $c$ are always observed to be rich $(a[30 / 0] c[30 / 0]), P_{r}(\operatorname{rich}(b))$ hits the highest value (1.0) while it decreases to less than one third $(0.28147)$ when $b$ 's friends are sometimes observed to be not

\footnotetext{
${ }^{24}$ For each observation set, we repeated parameter learning 100 times with random start and selected the parameter set that gave the highest likelihood. This applies to Table 2 as well.
} 
Table 2 MAP-learned probabilities with $a[0 / 30] c[0 / 30]$

\begin{tabular}{clll}
\hline$\alpha=\beta$ & $P_{r}(\operatorname{rich}(a))$ & $P_{r}(\operatorname{rich}(b))$ & $P_{r}(\operatorname{rich}(c))$ \\
\hline 1.01 & 0.00046 & 0.00013 & 0.00019 \\
1.1 & 0.00451 & 0.00170 & 0.00434 \\
2 & 0.02687 & 0.01338 & 0.01916 \\
10 & 0.13469 & 0.07649 & 0.12029 \\
100 & 0.52631 & 0.41646 & 0.52583 \\
1000 & 0.81027 & 0.72530 & 0.81030 \\
\hline
\end{tabular}

$\operatorname{rich}(a[20 / 10] c[10 / 20])$. When they are never observed to be rich $(a[0 / 30] c[0 / 30])$, it drops to 0.0 .

We notice that the last behavior, i.e. no observation means probability zero, is typical with MLE. If, however, one wishes to avoid this, it is possible to incorporate MAP (maximum a posteriori) estimation into the EMC algorithm though we do not detail it here. Actually when we applied a beta distribution prior $\alpha \theta^{\alpha-1}(1-\theta)^{\beta-1}$ uniformly to the probabilities of ground atoms and learned them with $a[0 / 30] c[0 / 30]$, changing $\alpha=\beta$ to $1.01,1.1,2,10$, 100 and 1000 respectively, $P_{r}(\operatorname{rich}(x))(x \in\{a, b, c\})$ always remained non-zero as shown in Table 2.

It is also interesting to see an interplay between logical inference and probabilistic inference. By logical inference we know $K B_{\text {rich }} \vdash \operatorname{rich}(a) \Leftarrow \operatorname{rich}(b)$. So $P_{r}(\operatorname{rich}(a) \Leftarrow$ $\operatorname{rich}(b))=1$ holds by the property of CBPMs. Consequently we have $P_{r}(\operatorname{rich}(a)) \geq$ $P_{r}(\operatorname{rich}(b))$, irrespective of learned parameters. Since the same holds for $c$, rich $(b)$ must satisfy two inequalities, $P_{r}(\operatorname{rich}(a)) \geq P_{r}(\operatorname{rich}(b))$ and $P_{r}(\operatorname{rich}(c)) \geq P_{r}(\operatorname{rich}(b))$, which certainly hold in Table 1 and in Table 2 for all learning cases.

\section{Related work}

To our knowledge, constraint-based probabilistic modeling is the first probabilistic modeling framework that uniformly deals with (discrete) log-linear models and rule-based probabilistic models. There is a large body of related work but we mention only some of them for reasons of space.

CFDs (case factor diagrams) (McAllester et al. 2004) define log-linear models at propositional level. A set of "feasible" truth assignments on (essentially) finitely many propositional variables are constructed by a CFD combining case statements and factor statements. A loglinear model is then defined considering each feasible assignment as a vector of Boolean features. CFDs and CBPMs are similar in that both define distributions over truth assignments. However CBPMs use first-order clauses instead of CFDs and can define a joint distribution over infinitely many propositional variables (ground atoms).

MLNs (Richardson and Domingos 2006) use typically first-order clauses like CBPMs but as feature functions to define log-linear models. What CBPMs differ most from (clausal) MLNs is the role of clauses. In CBPMs, unlike MLNs, clauses in a knowledge base $K B$ logically exclude Herbrand interpretations that falsify $K B$, giving them probability zero, and define a (possibly) non-uniform distribution over the remaining interpretations. In MLNs, the same effect is obtained by giving clauses equal weights and taking the infinite limit, but the resulting distribution is always uniform. Also we can simulate rule-based generative models such as PCFGs procedurally by CBPMs using iff $^{g}(R)$ as left-to-right rewriting rules 
(see Lemma 1 and Theorem 3) and calculate the probability of an observation from rule selection probabilities.

One of the salient features of CBPMs is the unconditional existence of probabilistic models in infinite domains (Theorem 4). Existing approaches are more or less conditional as far as we know. Probability measures (Gibbs measures) for infinite MLNs exist (Singla and Domingos 2007). Their existence however is guaranteed only when clauses are locally finite, in particular when they are $\sigma$-determinate, i.e. literals in a clause share the same set of variables over infinite domains. So clauses such as even $(x) \Leftarrow \operatorname{plus}(x, y, y)(x=y+y)$ in the natural number domain are prohibited. Similarly in the case of infinite BNs, there is some restriction (Pfeffer and Koller 2000; Kersting and De Raedt 2001; Milch et al. 2005; Laskey 2006). For example nodes cannot have infinite parents in recursive probability models (Pfeffer and Koller 2000). Bayesian logic programs (Kersting and De Raedt 2001) use Bayesian clauses of the form $A_{0} \mid A_{1}, \ldots, A_{n}$ to define a BN such that nodes are ground atoms in the least Herbrand model of the program and $A_{0}^{\prime}$ has incoming edges from $A_{1}^{\prime}, \ldots, A_{n}^{\prime}\left(A_{i}^{\prime}\right.$ is a ground instance of $A_{i}$ ). An infinite $\mathrm{BN}$ is definable under the condition that every node has finite ancestors and there is no cyclic path in the dependency graph derived from the program. In contingent BNs (Milch et al. 2005) where edges are labeled by events such as $X=0$, nodes can have infinite parents and cyclic paths are allowed. However the set of labels labeling a cyclic path, an infinite upward chain $X_{1} \leftarrow X_{2} \leftarrow \cdots$, and infinite incoming edges to a node must be inconsistent. MEBN (Laskey 2006) is a first-order probabilistic language based on MFrags which are schematic specifications of local BNs. For an infinite BN to be definable in MEBN, it is required that for each instantiated MFrag for a node, when parent nodes allowed by the MFrag are added indefinitely, the CPT stops changing at some point such that from that point on, no further addition of relevant parent nodes does not affect the CPT.

The EMC algorithm in Sect. 4 offers, though not always, an alternative parameter learning algorithm to the IM (iterative maximization) algorithm (Riezler 1998). The IM algorithm is applicable to log-linear models with incomplete data but since it solves numerical equations at every iteration say by Newton's method, it is a double loop algorithm. By comparison the EMC algorithm is a single-loop algorithm and simple to implement.

\section{Conclusion}

We have proposed constraint-based probabilistic modeling and proved that discrete probabilistic models (log-linear, rule-based) have an equivalent CBPM (constraint-based probabilistic model) $P_{c}(\cdot \mid K B)$ which is a joint distribution conditioned on a clausal set $K B$. We also proved the existence of CBPMs in infinite domains giving probability one to existentially closed logical consequences of $K B$. We then derived a new EM algorithm named the EMC algorithm applicable to log-linear models with hidden variables for the parameter learning of CBPMs. Finally we applied CBPMs to statistical abduction and proposed constraint-based statistical abduction that allows a knowledge base $K B$ to include arbitrary clauses unlike existing approaches.

Although we provided a theoretical basis for CBPMs in this paper, we have a long list of future research topics. They include Bayesian inference, approximate probability computation, computational complexity of CBPMs and applications to real data. Also the uniqueness condition of CBPMs in infinite domains and the treatment of graphical models with a mixture of directed and undirected edges are interesting future research topics. 
Acknowledgements We thank Dr. Yoshitaka Kameya for helpful discussion and careful reading of the manuscript. We also thank anonymous referees for their useful comments and suggestions.

\section{References}

Akers, S. (1978). Binary decision diagrams. IEEE Transactions on Computers, 27(6), 509-516.

Boutilier, C., Friedman, N., Goldszmidt, M., \& Koller, D. (1996). Context-specific independence in Bayesian networks. In Proceedings of the 12th conference on uncertainty in artificial intelligence (UAI'96) (pp. 115-123).

Bryant, R. (1986). Graph-based algorithms for boolean function manipulation. IEEE Transactions on Computers, 35(8), 677-691.

Chavira, M., \& Darwiche, A. (2005). Compiling Bayesian networks with local structure. In Proceedings of the 19th international joint conference on artificial intelligence (IJCAI'05) (pp. 1306-1312).

Chen, J., Muggleton, S., \& Santos, J. (2008). Learning probabilistic logic models from probabilistic examples. Machine Learning, 73, 55-85.

Chow, Y., \& Teicher, H. (1997). Probability theory (3rd ed.). Berlin: Springer.

Cussens, J. (2001). Parameter estimation in stochastic logic programs. Machine Learning, 44(3), $245-271$.

De Raedt, L., \& Kersting, K. (2008). Probabilistic inductive logic programming. In L. De Raedt, P. Frasconi, K. Kersting, \& S. Muggleton (Eds.), Probabilistic inductive logic programming-theory and applications. Lecture notes in computer science (pp. 1-27). Berlin: Springer.

De Raedt, L., Kimmig, A., \& Toivonen, H. (2007). ProbLog: a probabilistic Prolog and its application in link discovery. In Proceedings of the 20th international joint conference on artificial intelligence (IJCAI'07) (pp. 2468-2473).

Dempster, A. P., Laird, N. M., \& Rubin, D. B. (1977). Maximum likelihood from incomplete data via the EM algorithm. Journal of the Royal Statistical Society, Series B, 39(1), 1-38.

Feller, W. (1971). An introduction to probability theory and its applications. New York: Wiley.

Getoor, L., \& Taskar, B. (Eds.) (2007). Introduction to statistical relational learning. Cambridge: MIT Press.

Inoue, K., Sato, T., Ishihata, M., Kameya, Y., \& Nabeshima, H. (2009). Evaluating abductive hypotheses using an EM algorithm on BDDs. In Proceedings of the 21st international joint conference on artificial intelligence (IJCAI'09) (pp. 810-815).

Ishihata, M., Kameya, Y., Sato, T., \& Minato, S. (2008). Propositionalizing the EM algorithm by BDDs (Technical Report TR08-0004). Dept. of CS, Tokyo Institute of Technology.

Kersting, K., \& De Raedt, L. (2001). Adaptive Bayesian logic programs. In Proceedings of the 11th international conference on inductive logic programming (ILP'01) (pp. 104-117).

Lafferty, J., McCallum, A., \& Pereira, F. (2001). Conditional random fields: probabilistic models for segmenting and labeling sequence data. In Proceedings of the 18th international conference on machine learning (ICML'01) (pp. 282-289).

Laskey, K. (2006). MEBN: a logic for open-world probabilistic reasoning (C4I Center Technical Report C4I06-01). George Mason University Department of Systems Engineering and Operations Research.

Lloyd, J. W. (1984). Foundations of logic programming. Berlin: Springer.

McAllester, D., Collins, M., \& Pereira, F. (2004). Case-factor diagrams for structured probabilistic modeling. In Proceedings of the 20th annual conference on uncertainty in artificial intelligence (UAI'04) (pp. 382391). Arlington: AUAI.

Milch, B., Marthi, B., Sontag, D., Russell, S., Ong, D., \& Kolobov, A. (2005). Approximate inference for infinite contingent Bayesian networks. In Proceedings of the 10th international workshop on artificial intelligence and statistics (AISTATS'05) (pp. 1352-1359).

Minato, S. (2001). Zero-suppressed BDDs and their applications. International Journal on Software Tools for Technology Transfer, 3(2), 156-170.

Muggleton, S. (1996). Stochastic logic programs. In L. De Raedt (Ed.), Advances in inductive logic programming (pp. 254-264). Amsterdam: IOS.

Pfeffer, A., \& Koller, D. (2000). Semantics and inference for recursive probability models. In Proceedings of the 7th national conference on artificial intelligence (AAAI'O0) (pp. 538-544).

Poole, D. (1993). Probabilistic Horn abduction and Bayesian networks. Artificial Intelligence, 64(1), 81-129.

Poole, D. (1997). The independent choice logic for modeling multiple agents under uncertainty. Artificial Intelligence, 94(1-2), 7-56.

Poole, D. (2000). Abducing through negation as failure: stable models within independent choice logic. Journal of Logic Programming, 44, 5-35.

Richardson, M., \& Domingos, P. (2006). Markov logic networks. Machine Learning, 62, 107-136.

Riezler, S. (1998). Probabilistic constraint logic programming. $\mathrm{PhD}$ thesis, Universität Tübingen. 
Sato, T., \& Kameya, Y. (2001). Parameter learning of logic programs for symbolic-statistical modeling. Journal of Artificial Intelligence Research, 15, 391-454.

Sato, T., \& Kameya, Y. (2002). Statistical abduction with tabulation. In A. Kakas \& F. Sadri (Eds.), LNAI: Vol. 2408. Computational logic: logic programming and beyond (pp. 567-587). Berlin: Springer.

Sato, T., \& Kameya, Y. (2008). New advances in logic-based probabilistic modeling by PRISM. In L. De Raedt, P. Frasconi, K. Kersting, \& S. Muggleton (Eds.), LNAI: Vol. 4911. Probabilistic inductive logic programming (pp. 118-155). Berlin: Springer.

Singla, P., \& Domingos, P. (2007). Markov logic in infinite domains. In Proceedings of the twenty-third conference on uncertainty in artificial intelligence (UAI'07) (pp. 368-375). 